\title{
Development, Characterization, Stability and Bioaccessibility Improvement of 7,8-Dihydroxyflavone Loaded Zein/Sophorolipid/Polysaccharide Ternary Nanoparticles: Comparison of Sodium Alginate and Sodium Carboxymethyl Cellulose
}

\author{
Yufeng Chen ${ }^{1}{ }^{\mathbb{D}}$, Jingchong Peng ${ }^{1}$, Yueqi Wang ${ }^{2, *}$, Daniel Wadhawan ${ }^{3}$, Lijun Wu ${ }^{1}$, Xiaojing Gao ${ }^{1}$, Yi Sun ${ }^{1}$ \\ and Guobin Xia ${ }^{4, *}$
}

check for updates

Citation: Chen, Y.; Peng, J.; Wang, Y.; Wadhawan, D.; Wu, L.; Gao, X.; Sun,

Y.; Xia, G. Development,

Characterization, Stability and Bioaccessibility Improvement of 7,8-Dihydroxyflavone Loaded Zein/Sophorolipid/Polysaccharide Ternary Nanoparticles: Comparison of Sodium Alginate and Sodium Carboxymethyl Cellulose. Foods 2021, 10, 2629. https://doi.org/10.3390/ foods10112629

Academic Editors: Hong Wu and Hui Zhang

Received: 25 September 2021

Accepted: 25 October 2021

Published: 29 October 2021

Publisher's Note: MDPI stays neutral with regard to jurisdictional claims in published maps and institutional affiliations.

Copyright: (c) 2021 by the authors Licensee MDPI, Basel, Switzerland. This article is an open access article distributed under the terms and conditions of the Creative Commons Attribution (CC BY) license (https:// creativecommons.org/licenses/by/ $4.0 /)$
1 College of Food Science and Technology, Zhejiang University of Technology, Hangzhou 310014, China; wuyycyf@163.com (Y.C.); pjchs6g@163.com (J.P.); wulj416@163.com (L.W.); gaoxiaojing66@163.com (X.G.); sunyi971219@163.com (Y.S.)

2 Key Lab of Aquatic Product Processing, Ministry of Agriculture and Rural Affairs of the People's Republic of China, South China Sea Fisheries Research Institute, Chinese Academy of Fishery Sciences,

Guangzhou 510300, China

3 Department of Natural Science and Mathematics, University of Houston, Houston, TX 77002, USA; dkwadhawan2@uh.edu

4 Department of Pediatrics Section of Neonatology, Texas Children's Hospital, Houston, TX 77030, USA

* Correspondence: wangyueqi@scsfri.ac.cn (Y.W.); gxxia@texaschildrens.org (G.X.)

Abstract: In this study, two polysaccharides [sodium alginate (ALG) and sodium carboxymethyl cellulose (CMC)] were selected to establish zein/sophorolipid/ALG (ALG/S/Z) and zein/sophorolipid/ ALG (CMC/S/Z) nanoparticles to encapsulate 7,8-dihydroxyflavone (7,8-DHF), respectively. The results showed that polysaccharide types significantly affected performance of ternary nanoparticles, including $\mathrm{CMC} / \mathrm{S} / \mathrm{Z}$ possessed lower polydispersity index, particle size and turbidity, but higher zeta potential, encapsulation efficiency and loading capacity compared to ALG/S/Z. Compared to zein/sophorolipid nanoparticles (S/Z), both ALG/S/Z and CMC/S/Z had better stability against low pH (pH 3 4) and high ionic strengths (150 200 mM NaCl). Hydrophobic effects, electrostatic interactions and hydrogen bonding were confirmed in ternary nanoparticles fabrication via Fourier-transform infrared spectroscopy. Circular dichroism revealed that CMC and ALG had no evident impact on secondary structure of zein in $S / Z$, but changed surface morphology of $S / Z$ as observed by scanning electron microscope. Encapsulated 7,8-DHF exhibited an amorphous state in ternary nanoparticles as detected by $\mathrm{X}$-ray diffraction and differential scanning calorimetry. Furthermore, compared to $\mathrm{S} / \mathrm{Z}, \mathrm{ALG} / \mathrm{S} / \mathrm{Z}$, and $\mathrm{CMC} / \mathrm{S} / \mathrm{Z}$ remarkably improved the storage stability and bioaccessibility of 7,8-DHF. CMC/S/Z possessed a greater storage stability for 7,8-DHF, however, ALG/S/Z exhibited a better in vitro bioaccessibility of 7,8-DHF. This research provides a theoretical reference for zein-based delivery system application.

Keywords: Zein; 7,8-dihydroxyflavone; sophorolipid; polysaccharide; ternary nanoparticles

\section{Introduction}

7,8-dihydroxyflavone (7,8-DHF) (Figure S1), a naturally occurring infrequent flavone monomeric compound from Tridax procumbens and Godmania aesculifolia,, was authenticated as a high-affinity tropomyosin receptor kinase B (TrkB) agonist [1,2]. It could mimic the physiological roles of brain-derived neurotrophic factor (BDNF) and its associated downstream signaling pathway [3]. Current literature confirms that 7,8-DHF's role in attenuating many BDNF- relevant human illnesses, including Parkinson's disease, Alzheimer's disease, obesity, and depression [4-8]. However, glucuronidation, sulfation, and methylation of 
7,8-DHF in the intestinal tract or liver caused its extremely low oral bioavailability [9]. In our previous study, we testified that 7,8-DHF's permeability coefficient was lower than $3 \times 10^{-6} \mathrm{~cm} / \mathrm{s}$ and had an active efflux mediated by multidrug resistance-related proteins (MRPs, especially MRP 2 outflow) and P-glycoprotein (P-gp) [10]. Thus, the low chemical instability, poor water solubility, and intestinal efflux of 7,8-DHF limit its application as a nutraceutical ingredient in functional food and beverage products.

To resolve the previously mentioned limitations and challenges, constructing an effective delivery vehicle using food-grade polymer is a novel strategy for boosting the utilization of water-insoluble functional components, such as liposomes, microemulsions, nano-emulsions, nanosuspensions, and nanoparticles [11-14]. Among these carriers, protein-based polymers, such as ovalbumin [15], casein [16], gliadin [17], and zein [18], have been utilized to create colloidal delivery systems for phenolic compounds to improve their physicochemical stability, water insolubility, and bioaccessibility. Specifically, these polymers possess several benefits such as high levels of biocompatibility, biodegradability, and label-friendliness.

As a primary storage protein found in corn, zein occupies for approximately $45 \sim 50 \%$ of total protein content, and has a relatively high percentage of hydrophobic groups. Zein's high level of hydrophobic groups means that it is soluble in $60 \sim 95 \%$ ethanol-water solutions, but insoluble in water [19]. The varying solubility of zein indicates that zein colloidal delivery systems can be established by antisolvent precipitation (ASP) [20]. Nevertheless, when exposed to a certain temperature, ionic strength, and $\mathrm{pH}$ range, zein nanoparticles are highly susceptible to aggregation because of the strong hydrophobic attractions among them. Previous studies have shown that food-grade biopolymers and surfactants can improve the aggregation stability of zein nanoparticles by establishing a protective layer around them. Among these applications, zein-based ternary nanoparticles exhibited more advantages compared to binary nanoparticles. Dai et al. and Wei et al. report that zein/polysaccharide/surfactant ternary nanoparticles functioned as delivery systems for coenzyme $Q_{10}$ and curcumin, enhancing their structural stability and in vitro bioaccessibility [21,22]. Zhang et al. reported a one-step assembly method for building zein/caseinate/alginate nanoparticles, as propolis were successfully encapsulated in these carriers, their bioaccessibility was improved [23]. In addition, zein/caseinate/sodium alginate nanoparticles were also established to improve the controlled release and physicochemical properties of curcumin [24].

In our previous study, we utilized sophorolipid, a surfactant molecule, to function as fabrication stabilizer for zein binary nanoparticles. These nanoparticles displayed stability at varying salt concentrations $(0 \sim 100 \mathrm{mM} \mathrm{NaCl})$ and a wide $\mathrm{pH}$ range (5 9) [25]. However, at low $\mathrm{pH}$ conditions, their stability was low. Therefore, we aim to use biomacromolecules like polysaccharide in this study to construct zein ternary nanoparticles and solve the aforementioned defects. Sodium alginate (ALG) is a natural anionic polysaccharide with a structure of $(1 \rightarrow 4)$-b-d-mannan and $(1 \rightarrow 4)$-a-l-guluronopyranoys homopolymer sequence isolated from Phaeophyceae. ALG can be used as a carrier for biological food components [26]. In addition, sodium carboxymethyl cellulose (CMC) is also a suitable anionic polymer for the delivery of bioactive ingredients [27]. Due to their widespread availability and functional properties, CMC and ALG are favorable candidates for the protection of coated zein/sophorolipid binary nanoparticles when encapsulating 7,8-DHF.

The first objective of our research was to explore the effect of polysaccharides type (CMC and ALG) on the formation and performance of zein ternary nanoparticles. Subsequently, to improve in vitro bioaccessibility and storage stability of 7,8-DHF, zein ternary nanoparticles encapsulation were manufactured. Moreover, the microstructure and chemical structure of these ternary nanoparticles were analyzed by a series of characterization techniques. For precision diet intervention or functional food field, this research provides valuable insights to develop more effective delivery systems for 7,8-DHF, which will allow more 7,8-DHF to be absorbed by the human intestines, exerting greater biological effects for human heath. For functional beverage industry, stable zein-surfactant-polysaccharide deliv- 
ery systems allow hydrophobic active components, such as 7,8-DHF, to be better dispersed in the water-phase beverage system to develop more functional beverage products.

\section{Materials and Methods}

\subsection{Materials and Chemicals}

Pancreatin $(4 \times$ USP specification) and zein $(\geq 95 \%)$ and were bought from SigmaAldrich (Missouri, USA). 7,8-dihydroxyflavone ( $\geq 98 \%)$ was purchased from TCI Co., Ltd. (Tokyo, Japan). Sophorolipid was purchased from the Boliante Chemical Company (Xian, China). Sodium alginate $(>90 \%)$ was obtained from Macklin (Shanghai, China). Sodium carboxymethyl cellulose was purchased from Aladdin (Shanghai, China). Bile salts and pepsin (activity 3000 3500 $\mathrm{U} \mathrm{mg}^{-1}$ ) were obtained from Sangon Biotech Co., Ltd. (Shanghai, China). Other utilized reagents and chemicals were of analytical grade.

\subsection{Zein/Sophorolipid/Polysaccharide Ternary Nanoparticles Preparation}

Zein/sophorolipid/polysaccharide ternary nanoparticles and zein/sophorolipid binary nanoparticles $(\mathrm{S} / \mathrm{Z})$ were fabricated based on our previous study [25]. In detail, zein and sophorolipid (mass ratio 1:1, $w / w$ ) were both dissolved in $80 \%$ ethanol/water solution to prepare the stock solution ( $1 \%$ sophorolipid $+1 \%$ zein, $w / v)$. For preparation of zein/sophorolipid/polysaccharide ternary nanoparticles, the stock solution was quickly added into the polysaccharide (ALG or CMC) aqueous solution (antisolvent) in a volume ratio of 1:3 $(v / v)$ under continuous stirring at $800 \mathrm{rpm}$ for $30 \mathrm{~min}$. Subsequently, ethanol was removed by a rotary evaporator at appropriate temperature. The mass ratio $(w / w)$ of zein to polysaccharide was set to 30:1, 20:1, 10:1, 5:1, 3:1, 2:1, and 1:1, respectively. The prepared CMC-based ternary nanoparticles were expressed as CMC/S/Z 30:1, CMC/S/Z 20:1, CMC/S/Z 10:1, CMC/S/Z 5:1, CMC/S/Z 3:1, CMC/S/Z 2:1, and CMC/S/Z 1:1, respectively. And the ALG-based ternary nanoparticles were denominated as ALG/S/Z 30:1, ALG/S/Z 20:1, ALG/S/Z 10:1, ALG/S/Z 5:1, ALG/S/Z 3:1, ALG/S/Z 2:1, ALG/S/Z $1: 1$, respectively. In addition, the $S / Z$ was also fabricated by ASP methods; the difference was that antisolvent was deionized water without ALG or CMC. The final concentration of zein in every nanoparticle was $2.5 \mathrm{mg} / \mathrm{mL}$. Then, the $\mathrm{pH}$ value of each nanoparticle dispersion was adjusted to 4.0 for dispersions stability investigation. The CMC/S/Z 5:1 and ALG/S/Z 5:1 were used for the following structure characterization. The freshly prepared samples were stored at $4{ }^{\circ} \mathrm{C}$, and a portion of each sample was freeze-dried into lyophilized powder for the following testing and analysis.

\subsection{Polydispersity Index (PDI), Particle Size, Zeta Potential and Turbidity}

PDI, particle size, and zeta potential of fresh dispersions were characterized using a dynamic light scattering (DLS) instrument (Nano-ZS90 analyzer, Malvern, UK) at $25^{\circ} \mathrm{C}$. PDI and particle size was detected via light intensity at a fixed scattering angle of $90^{\circ}$, and the refractive index of water was set at 1.45. The zeta potential was calculated by Smoluchowski model. The turbidity of complex particles was tested at $600 \mathrm{~nm}$ using an ultraviolet-visible spectrophotometer at $25^{\circ} \mathrm{C}$.

\subsection{Physical Stability of Ternary Nanoparticles}

2.4.1. $\mathrm{pH}$ Influence

The influence of $\mathrm{pH}$ on physical stability of each nanoparticle dispersion was evaluated within a $\mathrm{pH}$ range of 3 to 9 adjusting by either $2 \mathrm{M} \mathrm{HCl}$ or $2 \mathrm{M} \mathrm{NaOH}$.

\subsubsection{Ionic Strength Influence}

Each nanoparticle dispersion was blended with $\mathrm{NaCl}$ to obtain samples with $0,25,50$,

100,150 and $200 \mathrm{mM} \mathrm{NaCl}$ concentrations and stored for $24 \mathrm{~h}$ for observing physical stability.

Size changes within nanoparticle dispersions were recorded using DLS at $25^{\circ} \mathrm{C}$. 


\subsection{Encapsulation of 7,8-DHF into Ternary Nanoparticles}

7,8-DHF encapsulation was conducted according to the methods described in Section 2.2. 7,8-DHF, sophorolipid, and zein were dissolved at a mass ratio of 1:10:10 and 1:5:5 in 80\% ethanol/water solution, respectively. The mass ratio of zein to polysaccharide was 5:1 in the final reaction system. 7,8-DHF encapsulation in binary and ternary nanoparticles were denoted as DHF-S/Z, DHF-CMC/S/Z, and DHF-ALG/S/Z. Loaded complex particles were reserved at $4{ }^{\circ} \mathrm{C}$, with other samples freeze-dried for $48 \mathrm{~h}$ to conduct further analysis.

\subsection{Encapsulation Efficiency (EE) and Loading Capacity (LC)}

The EE and LC of encapsulated 7,8-DHF were assessed by UPLC based on our previously described method [25]. Specifically, the nanoparticles were centrifuged at 10,000 $\times g$ for $10 \mathrm{~min}$ at $4{ }^{\circ} \mathrm{C}$ using a centrifugal machine (3K15, Sigma, Osterode, Germany). The supernatant (containing loaded 7,8-DHF) was removed and diluted 5-fold with methanol. And an equal volume of the initial suspension was diluted in 5-fold methanol to obtain initial 7,8-DHF. Then, EE and LC were calculated according to the following equation:

$$
\begin{gathered}
\text { EE }(\%)=\text { loaded 7,8-DHF /initial 7,8-DHF } \times 100 \\
\text { LC }(\%)=\text { loaded 7,8-DHF/weight of vehicle } \times 100
\end{gathered}
$$

\subsection{7,8-DHF Loaded Ternary Nanoparticles Characterization}

\subsubsection{Fourier-Transform Infrared (FTIR) Spectroscopy}

7,8-DHF and lyophilized sample under analysis were prepared by adding 99\% $\mathrm{KBr}$ disc and scanned on an FTIR spectrometer (Avatar 370, Nicolet, Madison, WI, USA). The spectral scanning range was $500 \sim 4000 \mathrm{~cm}^{-1}$ at a resolution of $4 \mathrm{~cm}^{-1}$. The analytical results were carried out by OMNIC software version 8.0.

\subsubsection{Circular Dichroism (CD) Spectrum}

Secondary structural characteristics of nanoparticle dispersions under analysis were measured using a CD spectrometer (J-1500, JASCO, Tokyo, Japan). The concentration of complex dispersions was $0.2 \mathrm{mg} / \mathrm{mL}$ corresponding to zein. The buffer in CD experiments was deionized water $(\mathrm{pH}=7)$. The secondary structure scanning region was 190 260 $\mathrm{nm}$ with a $0.1 \mathrm{~cm}$ path length. The bandwidth was $1.0 \mathrm{~nm}$ and scanning speed was $50 \mathrm{~nm} / \mathrm{min}$. The data were evaluated by Spectra Manager ${ }^{\mathrm{TM}}$ II Software equipped with CD spectrometer.

\subsubsection{Differential Scanning Calorimetry (DSC)}

Thermal behavior of 7,8-DHF and freeze-dried samples were studied via DSC (Mettler Toledo, Zurich, Switzerland). 2 10 mg of samples were accurately weighed and hermetically sealed in aluminum pans. An empty crucible under the same condition was used as a reference. Scanning calorimetry was performed from a range of 25 to $200{ }^{\circ} \mathrm{C}$ in N2 atmosphere at a heating rate of $10^{\circ} \mathrm{C} / \mathrm{min}$ under $30 \mathrm{~mL} / \mathrm{min}$ flow.

\subsubsection{X-ray Diffraction (XRD)}

The crystalline characteristic of lyophilized nanoparticles and selected 7,8-DHF were recorded on an X-ray diffractometer (Bruker D8, Karlsruhe, Germany). This diffractometer was carried at $40 \mathrm{kV}$ accelerating voltage and $40 \mathrm{~mA}$ tube current to produce copper $\mathrm{K} \alpha$ radiation. Soller slit and divergence slit were set at $2.5^{\circ}$ and $0.5^{\circ}$, respectively, the $2 \theta$ angle was ranged from $5^{\circ}$ to $90^{\circ}$.

\subsubsection{Transmission Electron Microscopy (TEM)}

10-fold diluted fresh nanoparticle dispersions were deposited on a copper grid with formvar-carbon coating. Then, the samples were air-dried for $5 \mathrm{~min}$ and stained with 
$2 \%$ uranyl acetate. TEM (JEM-1200 EX, Tokyo, Japan) was performed for microscopic observation at $120 \mathrm{kV}$ accelerating voltage.

\subsubsection{Field Emission Scanning Electron Microscope (FE-SEM)}

The surface morphology of polysaccharide samples and freeze-dried nanoparticles was captured by FE-SEM (GeminiSEM 300, ZEISS, Germany). Before analysis, 3-6 nm of a thick gold layer was covered on the sample surfaces. The electron microscope acceleration voltage was $15.0 \mathrm{kV}$.

\subsection{Storage Stability of 7,8-DHF}

7,8-DHF, DHF-S/Z, DHF-ALG/S/Z, and DHF-CMC/S/Z were performed at $5{ }^{\circ} \mathrm{C}$ for $72 \mathrm{~h}$ under dark and $25^{\circ} \mathrm{C}$ for 15 days under light, respectively. At an appropriate point in time, the samples were acquired with 7,8-DHF presence being measured by UPLC. The storage stability was calculated based on the following equation:

Retention rate $(\%)=$ retained 7,8-DHF concentration/initial 7,8-DHF concentration $\times 100$

\subsection{In Vitro Simulated Gastrointestinal Digestion}

Based on Yuan et al.'s study via some amendments [28], briefly, $10 \mathrm{~mL}$ of simulated gastric fluid (SGF, $3.2 \mathrm{mg} / \mathrm{mL}$ pepsin and $2 \mathrm{mg} / \mathrm{mL} \mathrm{NaCl}, \mathrm{pH}=2.5$ ) and $10 \mathrm{~mL}$ of 7,8DHF, DHF-S/Z, DHF-ALG/S/Z, and DHF-CMC/S/Z were mixed for incubating in a $37^{\circ} \mathrm{C}$ water bath shaker for $60 \mathrm{~min}$ at $100 \mathrm{rpm}$. After SGF digestion, $10 \mathrm{~mL}$ of abovementioned simulated gastric digestive fluids were rapidly adjusted to $\mathrm{pH} 7.4$ using 2 $\mathrm{M} \mathrm{NaOH}$. Whereafter, $10 \mathrm{~mL}$ of simulated intestinal fluid (SIF, $4 \mathrm{mg} / \mathrm{mL}$ pancreatin, 5 $\mathrm{mg} / \mathrm{mL}$ bile salts, $6.8 \mathrm{mg} / \mathrm{mL} \mathrm{K2HPO} 4$ and $8.8 \mathrm{mg} / \mathrm{mL} \mathrm{NaCl}, \mathrm{pH}=7.4$ ) was added into above-mentioned simulated gastric digestive fluids and incubated for $120 \mathrm{~min}$ at same temperature and revolving speed. Finally, the final digestive solution was centrifuged by $20,000 \times g$ centrifugal force for $1 \mathrm{~h}$, and the supernatant (the mixed micelle phase containing 7,8-DHF) was collected. The bioaccessibility (\%) was calculated based on the following equation:

Bioaccessibility $(\%)=7,8-\mathrm{DHF}$ concentration in the micelles phases $/ 7,8-\mathrm{DHF}$ concentration in the formulation $\times 100$

\subsection{Statistical Analysis}

Mean \pm SD was presented via at least three times for all data. One-way ANOVA followed by Tukey's honestly significant difference post hoc tests were utilized to assess potential significant differences among groups. A $p<0.05$ indicated significant differences between groups. Data analysis was carried out by Origin 2021 (Origin Lab Co., Northampton, MA, USA) and GraphPad Prism version 8.0 (GraphPad Sofware, San Diego, CA, USA).

\section{Results and Discussion}

\subsection{Effect of Polysaccharides on Ternary Nanoparticles Fabrication}

The effects of varying concentrations and types of polysaccharide on particle size of $\mathrm{S} / \mathrm{Z}$ were illustrated in Figure 1. S/Z was approximate $82.81 \mathrm{~nm}$ particle size. The particle size of the ternary nanoparticles was differentially altered from that of $S / Z$ after introduction with CMC and ALG into the binary system. After supplementation with $\mathrm{CMC}$, the particle size of $\mathrm{CMC} / \mathrm{S} / \mathrm{Z}$ stepwise grew as the concentration of $\mathrm{CMC}$ increased (Figure 1A). Moreover, the incorporation of ALG resulted in a significant increase in particle size $(p<0.05)$. And in comparison to CMC/S/Z, ALG/S/Z showed a greater particle size at the identical polysaccharide level (Figure 1B). The above phenomenon might be attributed to the distinctive molecular structure of two studied polysaccharides and interaction force among molecules. The changes in particle size of zein/sophorolipid/polysaccharide ternary nanoparticles are possibly a result of sufficient anionic CMC and ALG being ab- 
sorbed on the surface of $S / Z$ through the electrostatic attractions. The changes in PDI value of $\mathrm{CMC} / \mathrm{S} / \mathrm{Z}$ and $\mathrm{ALG} / \mathrm{S} / \mathrm{Z}$ was analogous to that of particle size. As the concentration of $\mathrm{CMC}$ and ALG increased, the PDI value of CMC/S/Z and ALG/S/Z gradually increased as well. However, ALG/S/Z showed a higher PDI value at the same polysaccharide level.

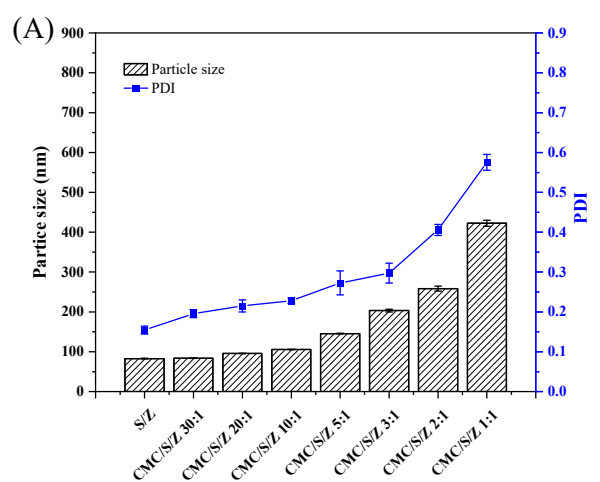

(B)
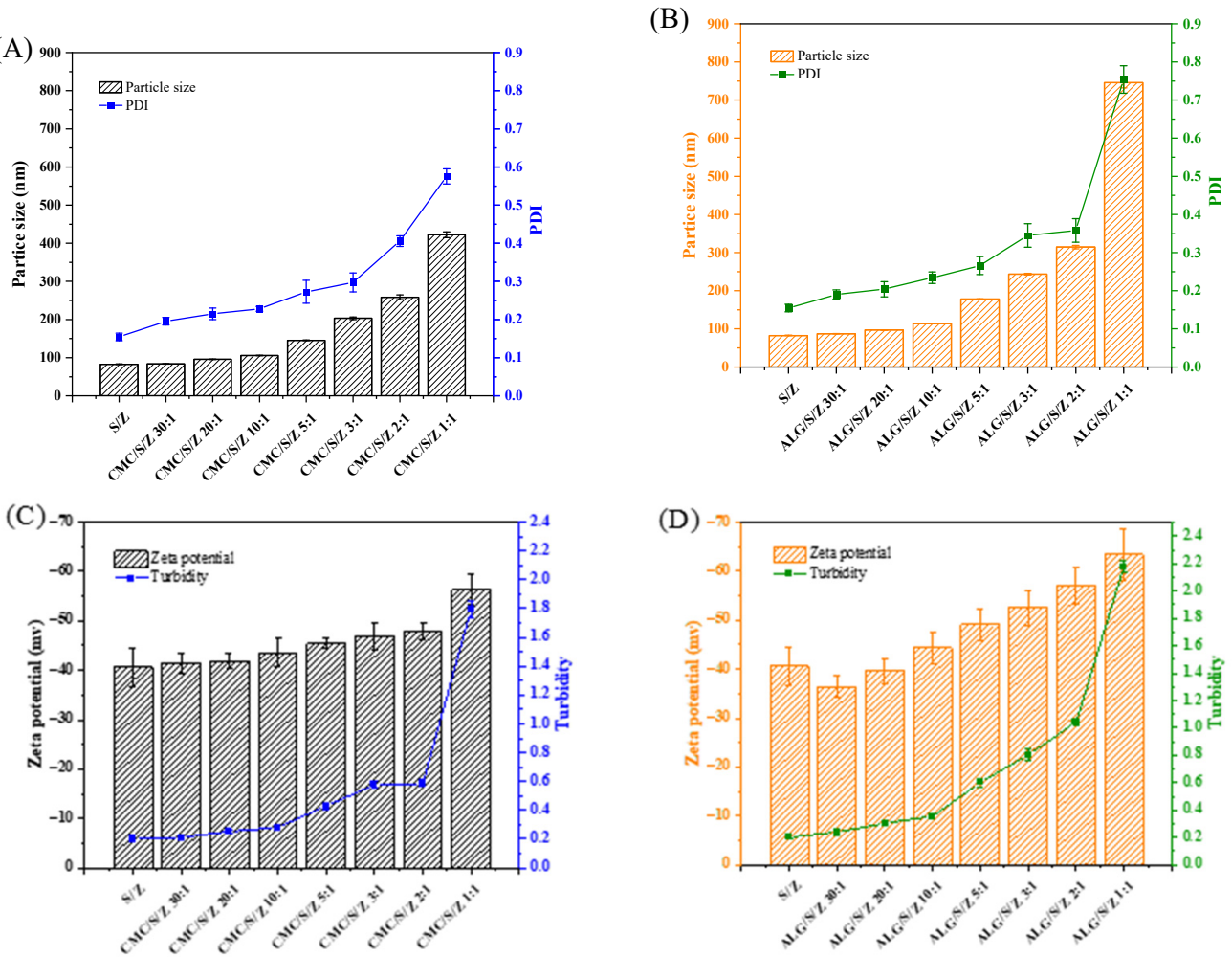

(E)

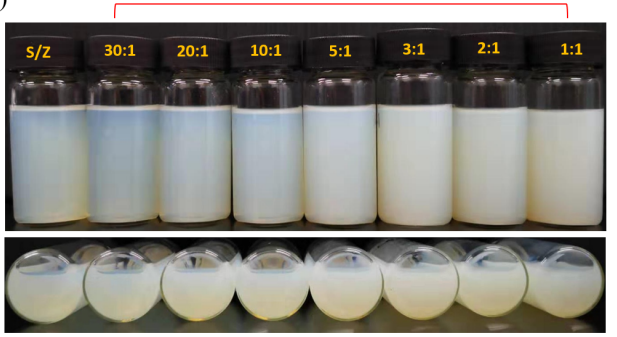

(F)

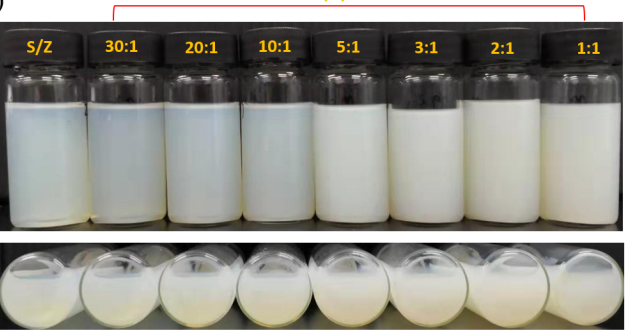

Figure 1. Particle size, PDI, zeta potential and turbidity of CMC/S/Z and ALG/S/Z with different zein to polysaccharide mass ratios. Particle size and PDI (A,B). Zeta-potential and turbidity (C,D). The photograph of each group appearance $(\mathbf{E}, \mathbf{F})$.

The zeta-potential of $\mathrm{S} / \mathrm{Z}$ was $-40.63 \mathrm{mV}$. When $\mathrm{CMC}$ and $\mathrm{S} / \mathrm{Z}$ were combined, their charge values became more negative as the fraction of present $C M C$ increased (Figure 1C). On the other hand, the negative charges of ALG/S/Z initially declined and grew whereafter when the level of ALG was increased (Figure 1D). When the mass ratio of polysaccharide to zein was above 1:10, the negative charges of $A L G / S / Z$ were higher than that of $\mathrm{CMC} / \mathrm{S} / \mathrm{Z}$. This difference is potentially caused by the different molecular structures of the two polysaccharides. The zeta-potential of zein/sophorolipid/polysaccharide ternary nanoparticles had been dominated by negatively charged CMC and ALG. It is reported that electrostatic attraction played a critical role in the formation of protein-surfactantpolysaccharide complexes, for instance, zein-rhamnolipid-propylene glycol alginate [29], and zein-lecithin-propylene glycol alginate [30]. Meanwhile, the uniform trend of turbidity in ternary nanoparticles was consistent with that of PDI value and particle size, as the increasing trend of turbidity was primarily caused by micro-aggregation of complexes [31]. 


\section{2. $C M C$ and $A L G$ Prevents $S / Z$ Ternary Nanoparticles Precipitation at $p H=4$}

Our previous work confirms that $\mathrm{S} / \mathrm{Z}$ exhibits weak stability in low $\mathrm{pH}$ conditions. To overcome this deficiency, we explore the influence of polysaccharide type (CMC and $\mathrm{ALG}$ ) and concentration on $\mathrm{S} / \mathrm{Z}$ stability at $\mathrm{pH}=4$. As shown in (Figure $2 \mathrm{~A}$ ), $\mathrm{S} / \mathrm{Z}$ ternary nanoparticles were extremely unstable and aggregated together as expected. This phenomenon could be elucidated by the lack of appropriate electrostatic repulsion, which is required to conquer attractive interactions (e.g., van der Waals) among nanoparticles. Furthermore, at low $\mathrm{pH}$ values, the $\mathrm{pKa}$ of hydrophilic sugar residues present on sophorolipid molecules were reported to promote this instability [32]. After CMC was added, the PDI value, particle size, and turbidity of $\mathrm{CMC} / \mathrm{S} / \mathrm{Z}$ initially declined and subsequently jumped until a mass of zein to $C M C$ was $5: 1$, the $C M C / S / Z$ were possessed the lowest values for particle size, $\mathrm{PDF}$, and turbidity (particle size $\approx 341.50 \mathrm{~nm}, \mathrm{PDI} \approx 0.335$ and turbidity $\approx 2.257$ ) (Figure $2 \mathrm{~A}, \mathrm{C}$ ). Moreover, the incorporation of ALG similarly decreased and then increased PDI value, particle size and turbidity of ALG/S/Z. The particle size for ALG/S/Z was smallest at the $10: 1(353.62 \mathrm{~nm})$, while the PDI value $(0.335)$ and turbidity (2.509) were lowest at 5:1 mass ratio of zein to ALG (Figure 2B,D). As seen from photograph (Figure 2E,F), no floccules were observed at the bottom of the container at varying zein to polysaccharide mass ratios (10:1, 5:1, 3:1, and 2:1). A reason for lacking floccules at these mass ratios might be a result of appropriate CMC and ALG participation, which potentially increased the steric or electrostatic repulsions for $\mathrm{S} / \mathrm{Z}$ against aggregation. Nevertheless, at a zein to polysaccharide mass of $1: 1$, there were sediments in both CMC/S/Z and ALG/S/Z. The underlying mechanism for these observations is bridging flocculation, i.e., the ability of a single CMC or ALG molecule to adsorb to the surfaces of two or more $\mathrm{S} / \mathrm{Z}$. Therefore, CMC or ALG adsorption results in the formation of clusters [33]. Furthermore, with the CMC and ALG concentrations raised, the charge values of CMC/S/Z and ALG/S/Z became increasingly negative. The intensification of these charges revalidates the idea that $\mathrm{CMC} / \mathrm{S} / \mathrm{Z}$ and $\mathrm{ALG} / \mathrm{S} / \mathrm{Z}$ experience sufficient electrostatic repulsion to avoid precipitation at varying mass ratios of 10:1 2:1. Based on these results, we subsequently selected CMC/S/Z and ALG/S/Z at a mass ratio of zein to polysaccharide at 5:1 as the optimum ratio to study in the following research.
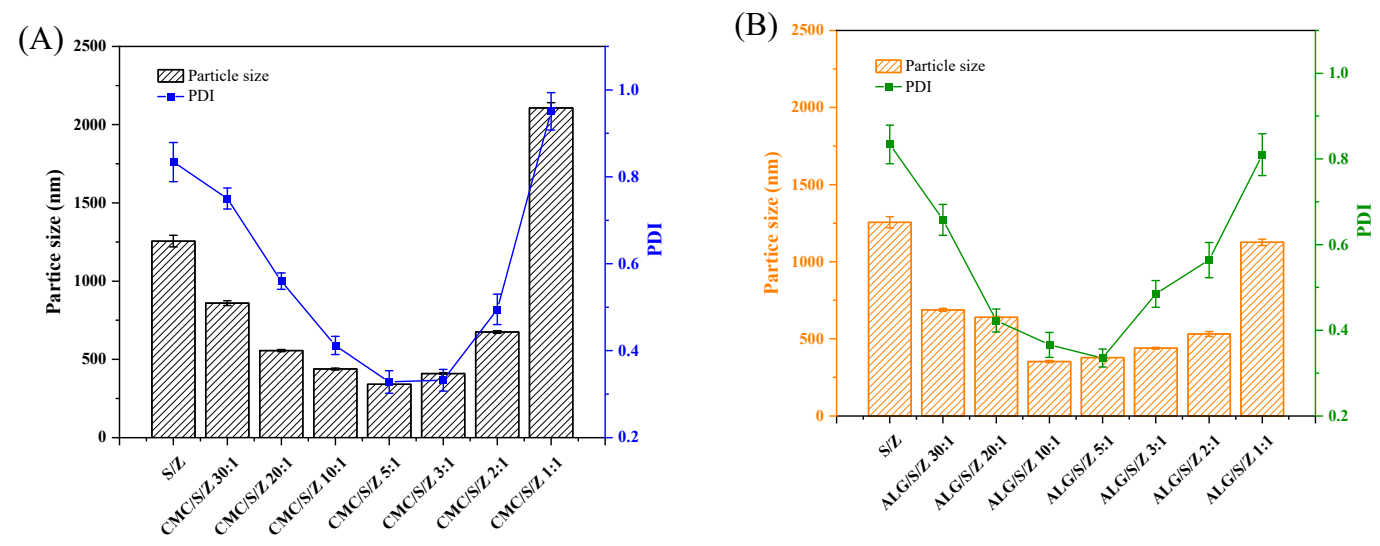

Figure 2. Cont. 


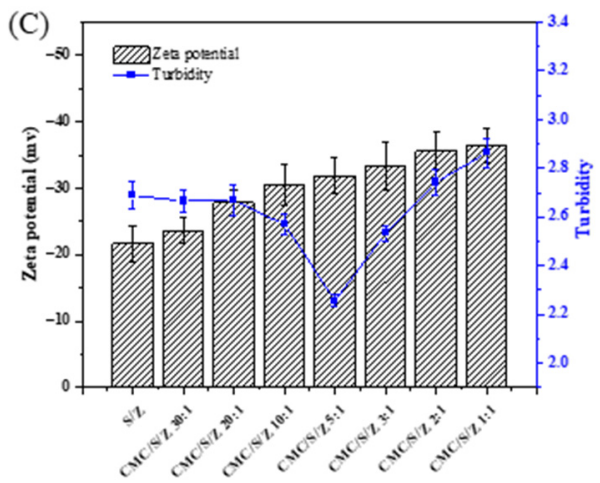

(E)

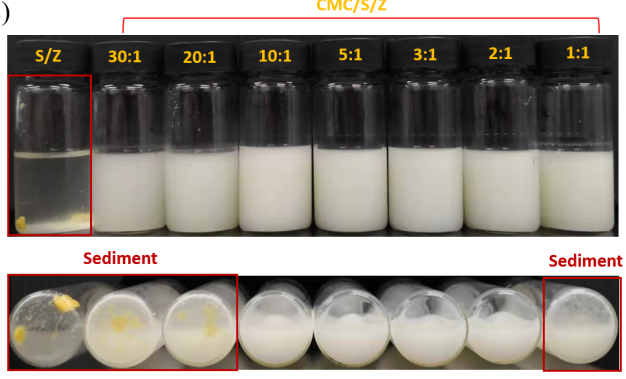

(D)

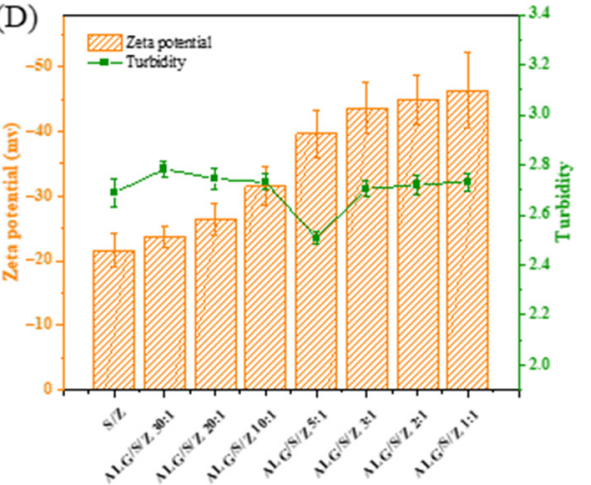

(F)

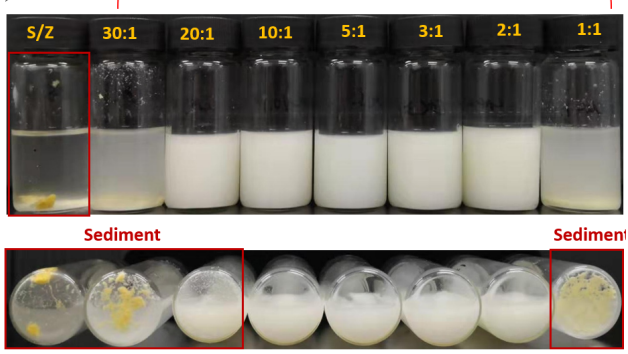

Figure 2. Particle size, PDI, zeta potential and turbidity of CMC/S/Z and ALG/S/Z with different zein to polysaccharide mass ratios at $\mathrm{pH}=4$. Particle size and PDI $(\mathbf{A}, \mathbf{B})$. Zeta-potential and turbidity $(\mathbf{C}, \mathbf{D})$. The photograph of each group appearance $(\mathbf{E}, \mathbf{F})$.

\subsection{Physical-Chemical Stability Study on Ternary Nanoparticles}

The optimum delivery system for food nutraceuticals must accommodate flexible $\mathrm{pH}$ and ionic environments during beverages processing, storage, and gut passage. Therefore, $\mathrm{pH}$ and salt stability testing of delivery systems is necessary to evaluate colloidal particles' functionality.

\subsubsection{Effect of $\mathrm{pH}$}

As seen from Figure $3 \mathrm{~A}, \mathrm{~S} / \mathrm{Z}$ exhibited extensive aggregation at $\mathrm{pH} 3.0$ and 4.0, exhibiting an increased particle size. This increase in size could be due to the fact that weakened electrostatic repulsion was weakened among binary nanoparticles. However, after adding CMC and ALG, compared to $S / Z$, the stability of both nanoparticles was enhanced noteworthily within $\mathrm{pH} 3.0$ to 4.0. Most notably, from $\mathrm{pH} 3$ to 9 , both $\mathrm{CMC} / \mathrm{S} / \mathrm{Z}$ and $\mathrm{ALG} / \mathrm{S} / \mathrm{Z}$ had excellent stability. It is reported that certain zein-surfactant or zein-polysaccharide binary nanoparticles, such as zein-rhamnolipid, zein-alginate, and zein-chondroitin sulfate, experienced a significant size increase at low $\mathrm{pH}$ levels [34-36]. Unexpectedly, zein-surfactant-polysaccharide ternary nanoparticles exhibited superior $\mathrm{pH}$ stability. There are some yellow floccules in the bottom of the container of $\mathrm{S} / \mathrm{Z}$ at $\mathrm{pH} 3.0$ and 4.0 but no floccules in CMC/S/Z and ALG/S/Z (Figure S4). 

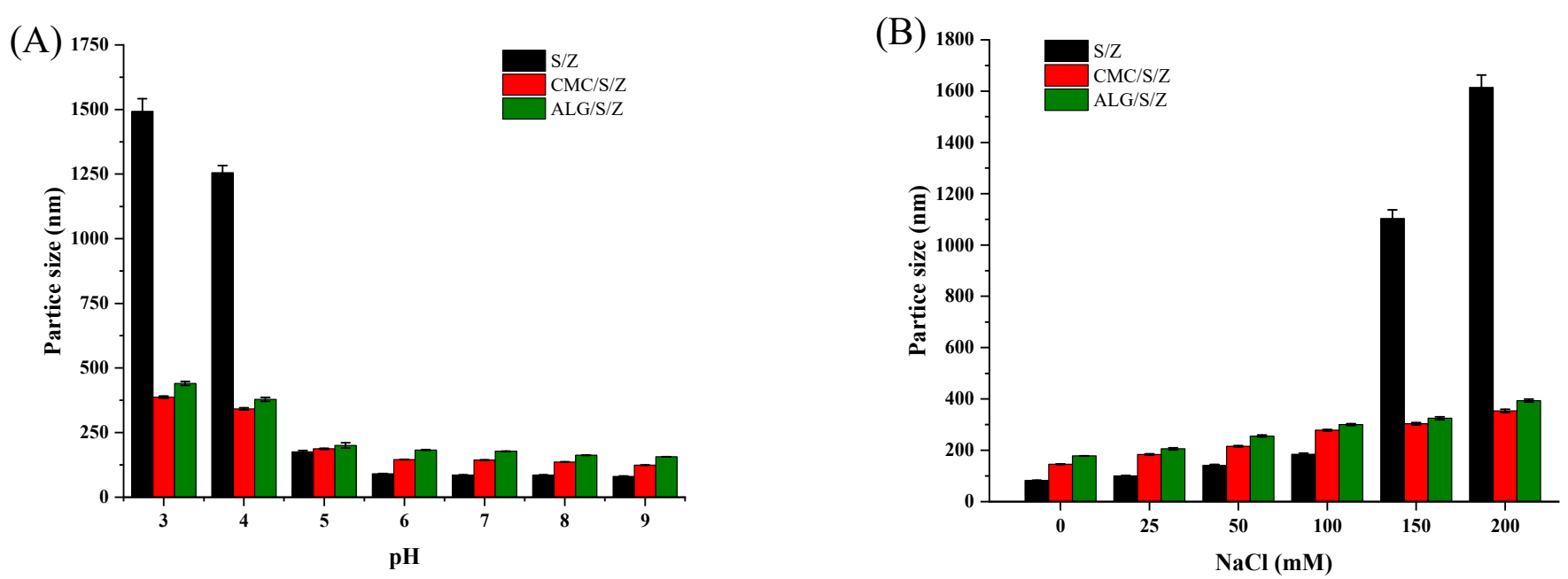

Figure 3. Particle size of colloidal particles at different $\mathrm{pH}$ conditions (A) and different $\mathrm{NaCl}$ concentrations (B).

\subsubsection{Effect of Ionic Strengths}

Particle size of S/Z was relatively low by 0 to $100 \mathrm{mM} \mathrm{NaCl}$ concentrations (Figure 3B). However, when increased to higher ionic strengths at $150 \sim 200 \mathrm{mM} \mathrm{NaCl}$ concentrations, precipitates were produced along with increasing particle size. This forming aggregation patterns for nanoparticles at high ionic strengths could be explained by increasing counterions (such as $\mathrm{Cl}^{-}$and $\mathrm{Na}^{+}$), depressed electrostatic repulsion via electrostatic screening and nanoparticle charge neutralization [21]. However, in the existence of CMC and ALG, the particle size of $\mathrm{CMC} / \mathrm{S} / \mathrm{Z}$ and $\mathrm{ALG} / \mathrm{S} / \mathrm{Z}$ was increased with the $\mathrm{NaCl}$ concentration increased. And adding CMC and ALG reduced the sensitivity of $S / Z$ to aggregation under high ionic strength condition. There are two possible reasons for the improved physical stability of CMC/S/Z and ALG/S/Z. Firstly, CMC and ALG prevented counterion neutralization and strong electrostatic repulsions, which could potentially restrain complex particles from coalescence via enhancing repulsive forces among particles. Secondly, an increase of the steric repulsion among particles could have occurred as polysaccharide molecules were attached to $\mathrm{S} / \mathrm{Z}$. Some floccules were also observed at the bottom of the tube for $\mathrm{S} / \mathrm{Z}$ at 150 and $200 \mathrm{mM} \mathrm{NaCl}$ (Figure S5). However, there were no floccules at the bottom of the tube for $\mathrm{CMC} / \mathrm{S} / \mathrm{Z}$ and $\mathrm{ALG} / \mathrm{S} / \mathrm{Z}$, in which stable colloidal systems were both developed.

\subsection{Encapsulation of 7,8-DHF}

The influence of polysaccharide types (CMC and ALG) and 7,8-DHF concentration on PDI value, particle size, EE and LC value in different delivery systems were summarized in Table 1. When the mass ratio of 7,8-DHF to zein was 1:5, the EE and LC of DHF-S/Z were 82.42 and $7.49 \%$, respectively. However, compared to DHF-S/Z, DHF-CMC/S/Z and DHF-ALG/S/Z exhibited better EE value, especially DHF-CMC/S/Z nanoparticles $(88.63 \%)(p<0.05)$. These findings suggested that polysaccharide addition improved the embedding ability of $S / Z$ via non-covalent interactions. There are two hydroxyl groups in the 7,8-DHF molecule that can interact with the hydroxyl groups of polysaccharides and the tyrosyl of zein. These interactions can form hydrogen bonds, possibly resulting in an increase of EE. These phenomena indicated that polysaccharides and S/Z showed a synergistic effect on the EE of 7,8-DHF. When mass ratio of 7,8-DHF to zein was 1:10, three colloidal delivery systems possessed high EE for 7,8-DHF (above 98.21\%). In contrast to unloaded colloidal delivery systems, the particle size of loaded colloidal delivery systems was growing when increasing the mass ratio of 7,8-DHF to zein. In general, 7,8-DHF, as a hydrophobic compound, is expected to be located within hydrophobic region of zein, ultimately possessing an impact on the size and stability of particles. 7,8-DHF encapsulation might impair interactions among hydrophobic groups of zein and increase particle size. 
Additionally, the encapsulation of 7,8-DHF caused an increase of PDI value in contrast to unloaded complexes. A uniform result was reported by Sun et al. [37]. The formation of free 7,8-DHF aggregates might help to explain the above observed phenomenon.

Table 1. EE, LC, particle size and PDI of 7,8-DHF in different colloidal systems.

\begin{tabular}{|c|c|c|c|c|c|}
\hline $\begin{array}{c}\text { Zein: } \\
7,8- \\
\text { DHF } \\
(w / w)\end{array}$ & $\begin{array}{l}\text { Colloidal } \\
\text { Systems }\end{array}$ & EE (\%) & LC (\%) & $\begin{array}{l}\text { Particle Size } \\
\text { (nm) }\end{array}$ & PDI \\
\hline \multicolumn{6}{|l|}{ Without } \\
\hline 7,8- & $S / Z$ & - & & $82.81 \pm 0.61^{\mathrm{a}}$ & $0.155 \pm 0.010^{a}$ \\
\hline \multicolumn{6}{|l|}{ DHF } \\
\hline & $\mathrm{CMC} / \mathrm{S} / \mathrm{Z}$ & - & & $145.6 \pm 0.75^{c}$ & $0.228 \pm 0.006^{b}$ \\
\hline & $\mathrm{ALG} / \mathrm{S} / \mathrm{Z}$ & - & & $178.2 \pm 0.35^{\mathrm{d}}$ & $0.266 \pm 0.024^{c}$ \\
\hline \multirow[t]{3}{*}{$5: 1$} & $\mathrm{~S} / \mathrm{Z}$ & $82.42 \pm 3.72^{a}$ & $7.49 \pm 0.22^{a}$ & $114.7 \pm 3.01^{b}$ & $0.271 \pm 0.016^{\mathrm{c}}$ \\
\hline & $\mathrm{CMC} / \mathrm{S} / \mathrm{Z}$ & $88.63 \pm 3.01^{b}$ & $7.38 \pm 0.13^{a}$ & $177.4 \pm 3.04^{\mathrm{a}}$ & $0.363 \pm 0.023 \mathrm{de}$ \\
\hline & $\mathrm{ALG} / \mathrm{S} / \mathrm{Z}$ & $84.15 \pm 2.63^{a}$ & $7.01 \pm 0.16^{a}$ & $214.3 \pm 3.21^{\mathrm{e}}$ & $0.394 \pm 0.022^{\mathrm{e}}$ \\
\hline \multirow[t]{3}{*}{$10: 1$} & $\mathrm{~S} / \mathrm{Z}$ & $98.21 \pm 1.31^{\mathrm{c}}$ & $4.68 \pm 0.40^{b}$ & $106.9 \pm 1.11^{b}$ & $0.201 \pm 0.021^{b}$ \\
\hline & $\mathrm{CMC} / \mathrm{S} / \mathrm{Z}$ & $99.51 \pm 0.24^{c}$ & $4.33 \pm 0.12^{b}$ & $168.4 \pm 3.62 \mathrm{dc}$ & $0.334 \pm 0.014^{\mathrm{d}}$ \\
\hline & ALG/S/Z & $98.71 \pm 1.12^{c}$ & $4.29 \pm 0.35^{b}$ & $200.1 \pm 2.01^{\mathrm{e}}$ & $0.352 \pm 0.017^{\mathrm{de}}$ \\
\hline
\end{tabular}

Values are the means \pm SD $(n=3)$. ${ }^{\mathrm{a}-\mathrm{e}}$ : Different letters in the same column indicate significant differences $(p<0.05)$ based on one-way ANOVA analysis followed by Tukey's honest significant difference post hoc tests.

\subsection{Characterization of Loaded Nanoparticles}

\subsubsection{FTIR}

FTIR is a versatile tool for monitoring changes within the functional groups of biopolymers and evaluating intermolecular interactions between components and particles. As shown in Figure 4A, the spectrum of CMC and ALG included diversiform representative carbohydrate peaks. The broad 3446 and $3441 \mathrm{~cm}^{-1}$ peaks represented hydroxyl groups $(\mathrm{O}-\mathrm{H})$ stretching, and the sharp 1604 and $1615 \mathrm{~cm}^{-1}$ peaks are associated with carboxyl (-COO-) symmetrical stretching vibration [38]. The featured peaks of CMC and ALG spectra appear at 1418 and $1417 \mathrm{~cm}^{-1}$, corresponding to rhamnogalacturonan moiety [39]. Multiple simultaneous vibration peaks in CMC and ALG at $900 \sim 1350 \mathrm{~cm}^{-1}$ were accredited to characteristic peaks of polysaccharides [24]. In our previous study, we confirmed that there are electrostatic attractions, hydrophobic interactions, and hydrogen bonding in $\mathrm{S} / \mathrm{Z}$. The FTIR spectrum of S/Z nanoparticles showed typical characteristic peaks at 3368, 1657 , and $1545 \mathrm{~cm}^{-1}$, respectively. When CMC and ALG were added, the peaks of O-H stretching vibration (3100 3500 $\mathrm{cm}^{-1}$ peaks) shifted from 3368 (S/Z) to 3382 (CMC/S/Z) and $3402 \mathrm{~cm}^{-1}$ (ALG/S/Z) [40]. This characteristic peak migration implied that there was a strong hydrogen bond between - $\mathrm{OH}$ groups in polysaccharides and amide group of glutamine in zein [41]. According to Liu et al.'s report [42], the $1657 \mathrm{~cm}^{-1}$ peak of zein at was the $\mathrm{C}=\mathrm{O}$ stretching (amide I). $1545 \mathrm{~cm}^{-1}$ peak was primarily associated with bending of N-H coupled with the stretching of C-N (amide II). With CMC and ALG incorporation, the amide I and amide II characteristic peaks of ternary nanoparticles were switched to $\left(1650\right.$ and $\left.1651 \mathrm{~cm}^{-1}\right)$ and $\left(1544\right.$ and $\left.1544 \mathrm{~cm}^{-1}\right)$, respectively. These results revealed electrostatic attractions were related to the establishment process of $\mathrm{CMC} / \mathrm{S} / \mathrm{Z}$ and ALG/S/Z. Based on these results, we confirmed that ALG/S/Z possessed stronger hydrogen bonding and electrostatic attraction than $\mathrm{CMC} / \mathrm{S} / \mathrm{Z}$. As seen from Figure $4 \mathrm{~B}$, the peaks at $3114,1626,1575,1405,1195$, and $1071 \mathrm{~cm}^{-1}$ were the typical peaks of 7,8-DHF, which have been confirmed in our previous study $[25,43]$. Expectedly, these characteristic peaks of 7,8-DHF were vanished in both binary and ternary nanoparticles, indicating that DHF-S/Z, DHF-CMC/S/Z, and DHF-ALG/S/Z samples successfully encapsulated for 7,8-DHF. 

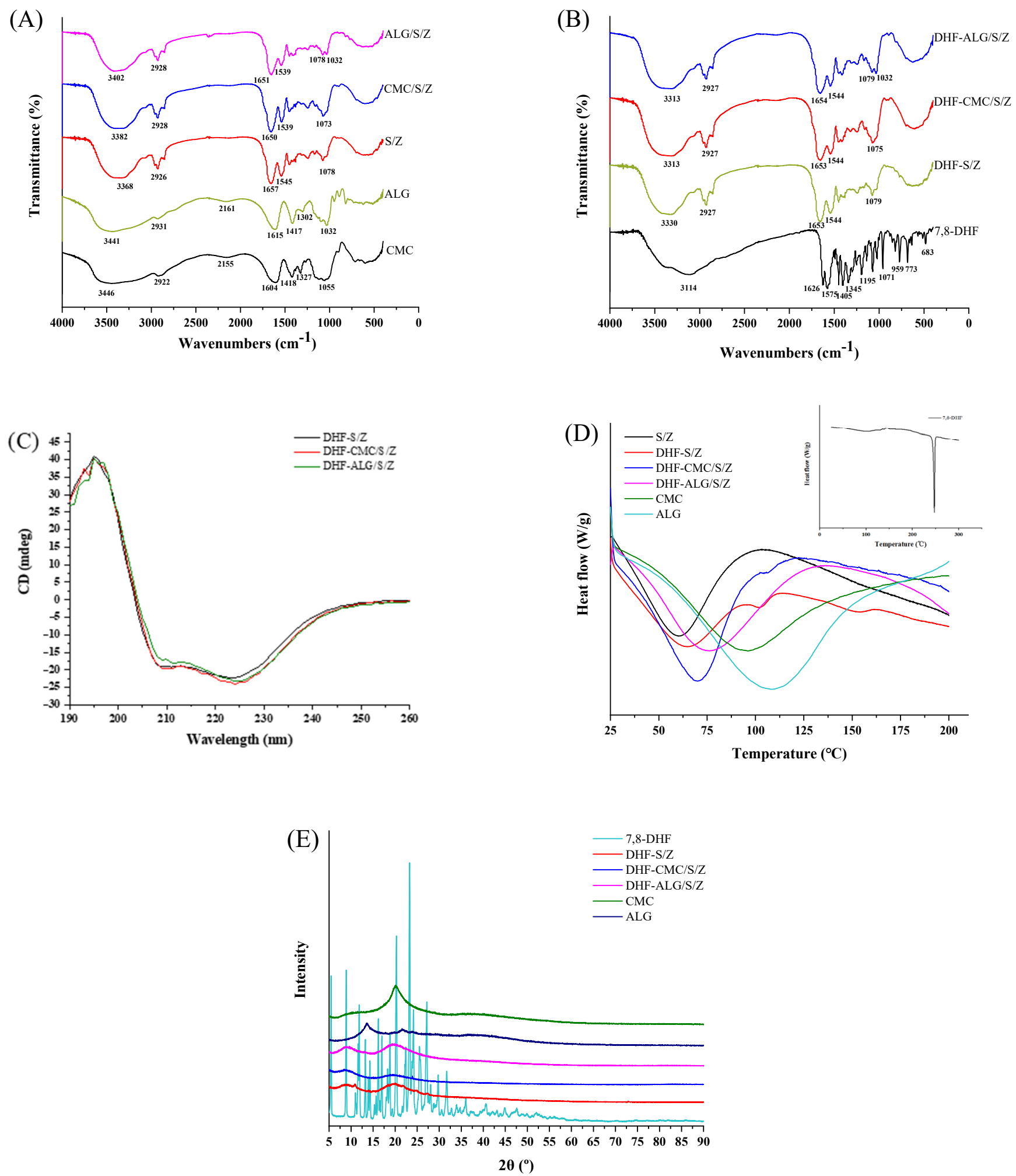

Figure 4. FTIR spectra of CMC, ALG and bare complex nanoparticles (A), free 7,8-DHF and 7,8-DHF loaded in each complex nanoparticles (B), CD spectra of zein in DHF-Z, DHF-Z/S-CMC, and DHF-ALG/S/Z (C), DSC analysis of free 7,8-DHF, CMC, ALG, S/Z, and 7,8-DHF loaded each complex nanoparticles (D), XRD spectra of free 7,8-DHF, polysaccharides and loaded each complex nanoparticles $(\mathrm{E})$. 


\subsubsection{Spectrum}

In this study, $\mathrm{CD}$ spectrum analysis was applied to measure conformational changes (secondary structure) of zein in complexation $(260 \mathrm{~nm} \sim 190 \mathrm{~nm}$ ). As seen from (Figure 4C), two peaks at 209 and $223 \mathrm{~nm}$ with a zero-crossing were around $203 \mathrm{~nm}$ (typical secondary structure) in the zein spectrum [44]. As shown in (Table 2), the $\alpha$-helix, $\beta$-sheet and $\beta$-turn content of DHF-S/Z changed from 25.3 to 24.7 and $24.5 \%, 25.8$ to 26.1 and $26.6 \%$ and 28.3 to 29.1 and $27.9 \%$, respectively after adding CMC and ALG. These results suggested that the addition of CMC and ALG had no apparent impact on zein's secondary structure in DHF-S/Z. The behavior of CMC and ALG could possibly be the result of sophorolipid adsorption on the zein surface. This adsorption could have possibly resulted in insufficient contact between the polysaccharides and zein, which was difficult to further change the secondary structure of zein. In general, the amide I band shifts in FTIR spectra could also reflect secondary structure changes of the protein, including the band of $\alpha$-helix, $\beta$-sheet, and $\beta$-turn [45]. In the FTIR spectra of our study (Figure $4 B$ ), the band of amide I did not show significant change among DHF-S/Z, DHF-CMC/S/Z and DHF-ALG/S/Z. This lack of significant change further confirmed that CMC and ALG did not impact the secondary structure of zein.

Table 2. Secondary structure of zein in DHF-S/Z, DHF-CMC/S/Z and DHF-ALG/S/Z.

\begin{tabular}{ccccc}
\hline \multirow{2}{*}{ Sample } & \multicolumn{4}{c}{ Content (\%) } \\
\cline { 2 - 5 } & $\boldsymbol{\alpha}$-Helix & $\beta$-Sheet & $\beta$-Turns & Unordered \\
\hline DHF-S/Z & $25.3 \pm 0.23$ & $25.8 \pm 0.21$ & $20.6 \pm 0.19$ & $28.3 \pm 0.26$ \\
DHF-CMC/S/Z & $24.7 \pm 0.18$ & $26.1 \pm 0.17$ & $20.1 \pm 0.21$ & $29.1 \pm 0.20$ \\
DHF-ALG/S/Z & $24.5 \pm 0.16$ & $26.6 \pm 0.23$ & $21.0 \pm 0.26$ & $27.9 \pm 0.24$ \\
\hline
\end{tabular}

\subsubsection{DSC}

Thermal properties of individual components and composite nanoparticles were studied via DSC. As shown in (Figure 4D), the embedded thermograms of 7,8-DHF displayed a narrow and sharp peak at $246.24{ }^{\circ} \mathrm{C}$. This temperature peak was probably caused by the melting of 7,8-DHF crystals [46]. Furthermore, the representative endothermal peak of CMC and ALG was at approximately 96.33 and $113.33^{\circ} \mathrm{C}$, respectively. These findings confirmed that ALG had a higher thermostability than CMC because of its specific carbohydrate structure. However, the endothermic peak of $S / Z$ was at approximately $60.33^{\circ} \mathrm{C}$, showing a low thermostability. After 7,8-DHF was encapsulated, no endothermic peaks of 7,8-DHF were found in DHF-S/Z, DHF-CMC/S/Z, and DHF-ALG/S/Z. The lack of endothermic peaks verified that 7,8-DHF was defined as an amorphous form rather than a crystalline form. Similar literature has reported in recent study on curcumin [47] and hyperoside [48]. In addition, the endothermic peak of DHF-S/Z nanoparticles was increased from $60.33{ }^{\circ} \mathrm{C}$ to $64.66{ }^{\circ} \mathrm{C}$ compared to $\mathrm{S} / \mathrm{Z}$. This increase in melting temperatures could be attributed to intermolecular interactions among 7,8-DHF, zein and sophorolipids [29]. Most importantly, after adding CMC and ALG, the endothermic peak of DHF-CMC/S/Z and DHF-ALG/S/Z rose to 70.01 and $76.33^{\circ} \mathrm{C}$ in comparison to DHF-S/Z, respectively. The higher endothermic peak of DHF-CMC/S/Z and DHF-ALG/S/Z manifested that they possessed better thermal stability than DHF-S/Z. This new peak might be the result of CMC and ALG interaction enhancements for hydrophobic, electrostatic, or hydrogen bond interactions among different components in nanoparticles, which ultimately leads to a higher endothermic peak temperature.

\subsubsection{XRD}

X-ray diffraction ranging from $5^{\circ}$ to $90^{\circ}$ at $2 \theta$ values was applied to examine $7,8-\mathrm{DHF}^{\prime} \mathrm{s}$ physical state in different nanoparticles. As presented in (Figure 4E), 7,8-DHF was highly crystalline with multiple sharp diffraction peaks at the range of $5 \sim 40^{\circ}$. On the one hand, when the diffraction angles of ALG in the $2 \theta$ range were $13.2^{\circ}$ and $21.8^{\circ}$, two flat peaks 
without sharp diffraction maximum appeared in the XRD spectrum. On the other hand, CMC only had a flat peak at a diffraction angle of $20.2^{\circ}$, while no sharp diffraction peaks were emerged. This XRD hump indicated that both pure polysaccharides were totally in an amorphous form [21,32]. However, no obvious sharp diffraction peaks about crystalline form for 7,8-DHF were found in both binary and ternary nanoparticles. Such behavior indicated 7,8-DHF was completely loaded into nanoparticles with an amorphous state. Interestingly, in comparison to DHF-S/Z, the hump for DHF-ALG/S/Z was distinctly increased, while the hump of DHF-CMC/S/Z was only slightly increased as the diffraction angle was at $19.6^{\circ}$. Thus, the polysaccharide types applied to construct nanocomposites can significantly affect XRD patterns. Moreover, no characteristic peaks for these two polysaccharides were observed. Collectively, due to changes in the interactions (hydrophobic, hydrogen bonding, and electrostatic) among zein, polysaccharide, and surfactant molecule, distinguishable behaviors of 7,8-DHF in DHF-S/Z, DHF-CMC/S/Z, and DHF-ALG/S/Z were observed.

\subsection{Micromorphology}

The microstructural features of loaded composite nanoparticles were analyzed via TEM. As observed in (Figure 5A), the diameter of DHF-S/Z was roughly $100 \mathrm{~nm}$, this finding was in agreement with the results of dynamic light scattering. After adding $C M C$ and ALG, DHF-CMC/S/Z and DHF-ALG/S/Z showed a similar spherical structure that was comparable to the DHF-S/Z. However, the diameter of DHF-CMC/S/Z and DHFALG/S/Z was larger than that of DHF-S/Z, exhibiting a particle size of 100 200 nm (Figure $5 B, C$ ). These findings demonstrate that CMC and ALG were absorbed on the surface of DHF-S/Z. Additionally, the diameter of DHF-CMC/S/Z was larger than that of DHF-ALG/S/Z, which was consistent with the results of DLS.
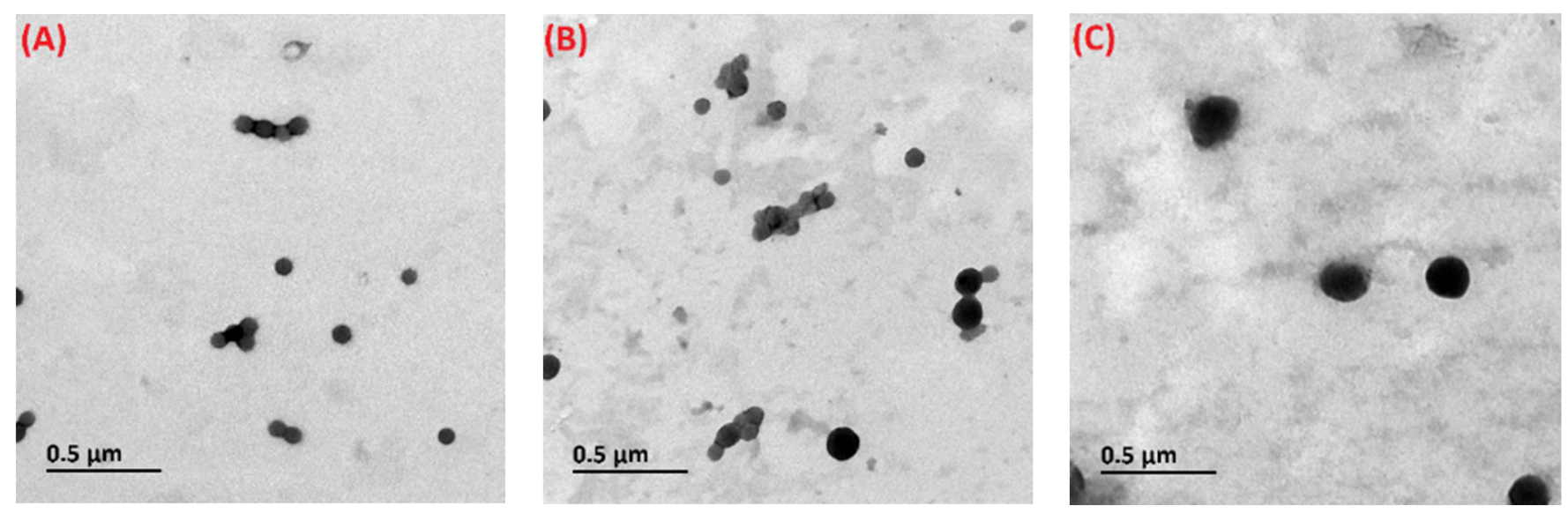

Figure 5. TEM images of DHF-S/Z (A), DHF-CMC/S/Z (B) and DHF-ALG/S/Z (C), 30,000× magnification times.

Furthermore, FE-SEM was applied to further observe the differences in surface microscopic morphology among individual composite nanoparticles (Figure 6). As seen from (Figure 6A), the surface morphology of DHF-S/Z expressed an irregular and rough shape consisting of many interlinked independent complexes. This morphological change was possibly attributed to sophorolipid adsorption on the surface of zein particles. The microphotograph of CMC and ALG depicted a shape similar to that of a silk ribbon (Figure $6 \mathrm{D}, \mathrm{E}$ ). When CMC and ALG were incorporated into DHF-S/Z, the micromorphology of DHF-CMC/S/Z and DHF-ALG/S/Z were spherical with uniform size and smooth surface (Figure 6B,C). The particle sizes of DHF-CMC/S/Z and DHF-ALG/S/Z were larger than that of DHF-S/Z. The polysaccharide coating on the outer layer of DHF$\mathrm{S} / \mathrm{Z}$ nanoparticles via electrostatic attraction possibly caused this size difference [49]. The above results were consistent with DLS measurements. 

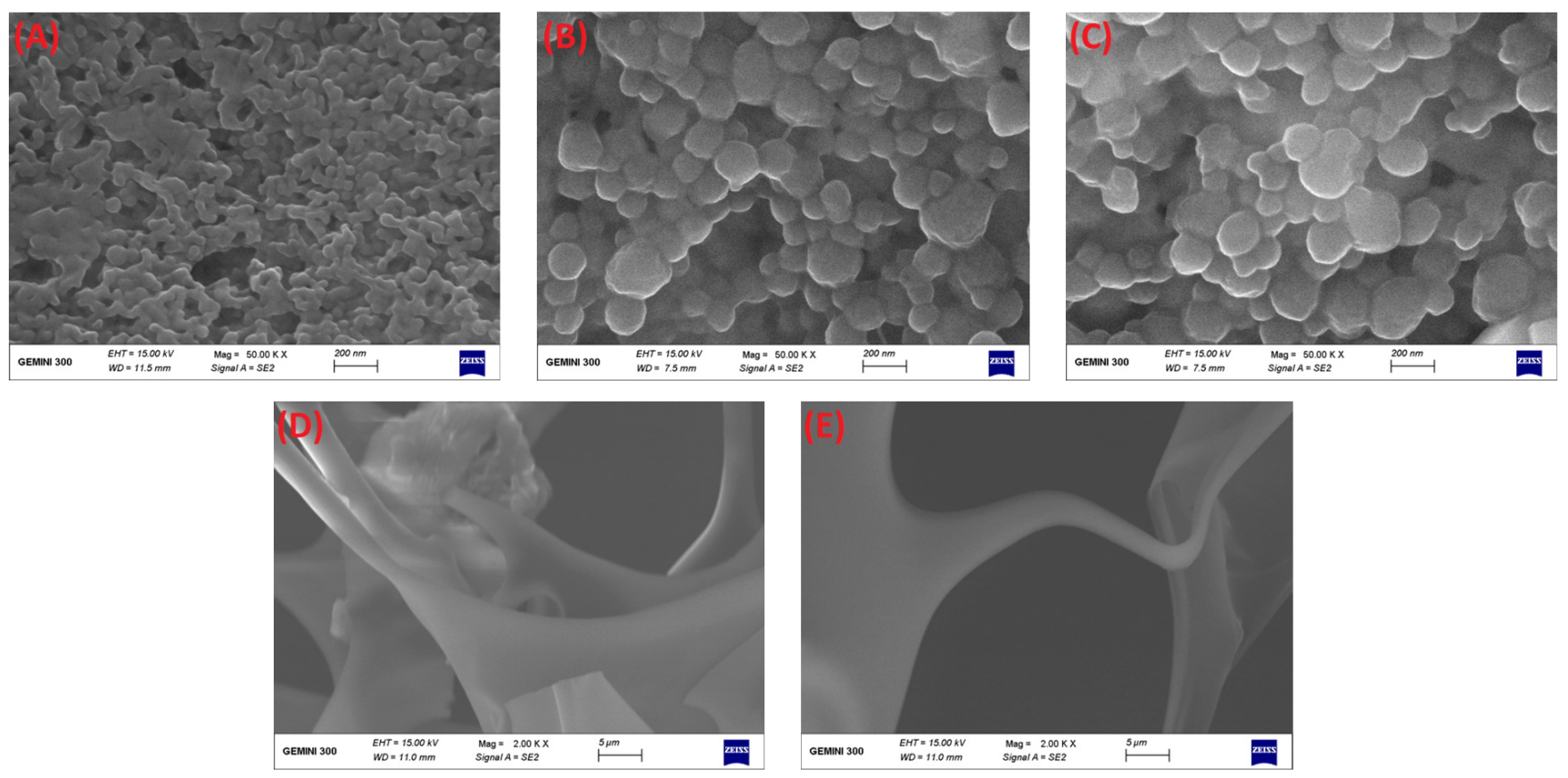

Figure 6. FE-SEM images of DHF-S/Z (A), DHF-CMC/S/Z (B), DHF-ALG/S/Z (C) (50,000× magnification times), individual CMC (D) and ALG (E) $(2000 \times$ magnification times).

\subsection{A Graphic Illustration for the Formation and Stability Mechanism of Nanoparticles}

Diverse technologies and measurements including EE, PDI, particle size, zeta potential, turbidity, CD, TEM, FE-SEM, DSC, XRD, and FTIR were used to make clear the formation and stability mechanism of zein/sophorolipid/polysaccharide ternary delivery system (Figure 7). After zein and sophorolipid were rapidly added into polysaccharide (polysaccharide: zein mass ratio $\leq 1: 2$ ), sufficient CMC or ALG acted as a shielding effect (electrostatic repulsion) and steric hindrance stabilizer was coated onto the surface of $\mathrm{S} / \mathrm{Z}$ particles to prevent their sedimentation at low $\mathrm{pH} 3 \sim 4$ range condition, showing a homogeneous PDI, turbidity and size based on DLS and UV. Certain internal drives (electrostatic interaction, hydrogen bonding and hydrophobic effect) participated in the formation of DHF-CMC/S/Z and DHF-ALG/S/Z, and CMC and ALG had no significant impact on secondary structure of zein in S/Z according to CD, FTIR, and FE-SEM. Upon employing EE, XRD, DSC, TEM analysis, 7,8-DHF was shown to be successfully encapsulated in CMC/S/Z and ALG/S/Z with relatively uniform sphericity, displaying a good entrapment efficiency. 


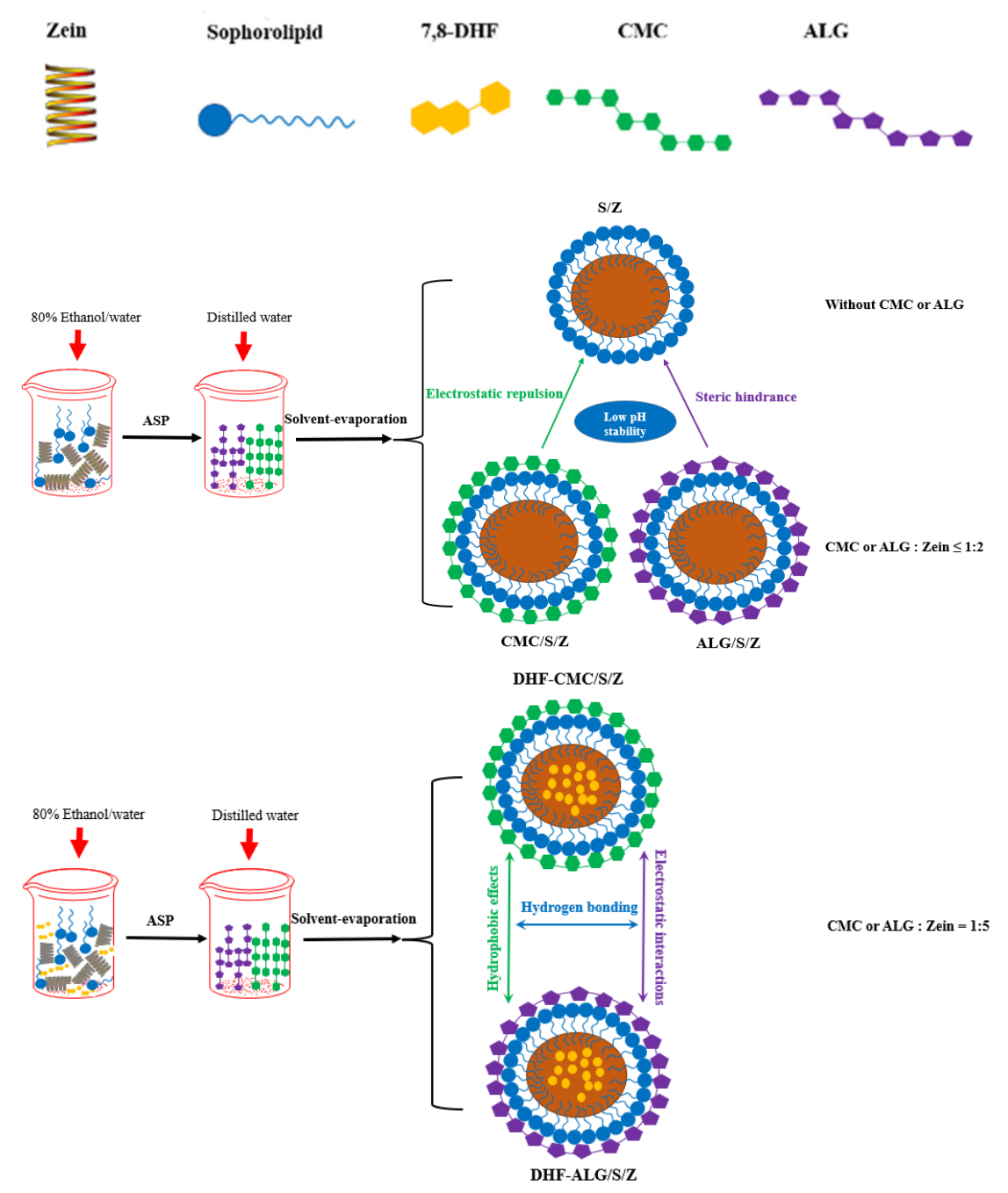

Figure 7. An illustration of the formation and stability mechanism of DHF-CMC/S/Z and DHF$\mathrm{ALG} / \mathrm{S} / \mathrm{Z}$.

\subsection{Storage Stability of 7,8-DHF}

During storage, preventing food neutraceuticals from heat or light exposure is challenging, but critically necessary for mitigating degradation. To meet this application end, short and long term storage were investigated under varying environments for 7,8-DHF, loaded binary and ternary nanoparticles. As shown in (Figure 8A), free 7,8-DHF was mostly degraded at $25{ }^{\circ} \mathrm{C}$ with light exposure for 15 days post-storage. Encapsulation of the 7,8-DHF in S/Z nanoparticles strengthened storage stability of 7,8-DHF (26.26\%). The addition of CMC and ALG further enhanced the stability of encapsulated 7,8-DHF, in particular DHF-CMC/S/Z (58.75\%). At $50{ }^{\circ} \mathrm{C}$ under dark conditions (Figure $8 \mathrm{~B}$ ), a similar effect was observed. The active groups of 7,8 -DHF were possibly protected within the hydrophobic lumen of DHF-S/Z, DHF-CMC/S/Z, and DHF-ALG/S/Z nanoparticles as a mechanism [50], besides, due to the different chemical structures of CMC and ALG, DHF$\mathrm{CMC} / \mathrm{S} / \mathrm{Z}$ have higher EE, which caused more 7,8-DHF to be protected in the hydrophobic part. These results are in agreement with previous studies that introduced curcumin being embedded in zein and quaternized chitosan complexes [51], along with work showing that quercetagetin was loaded using the zein-hyaluronic acid binary complexes [52]. 

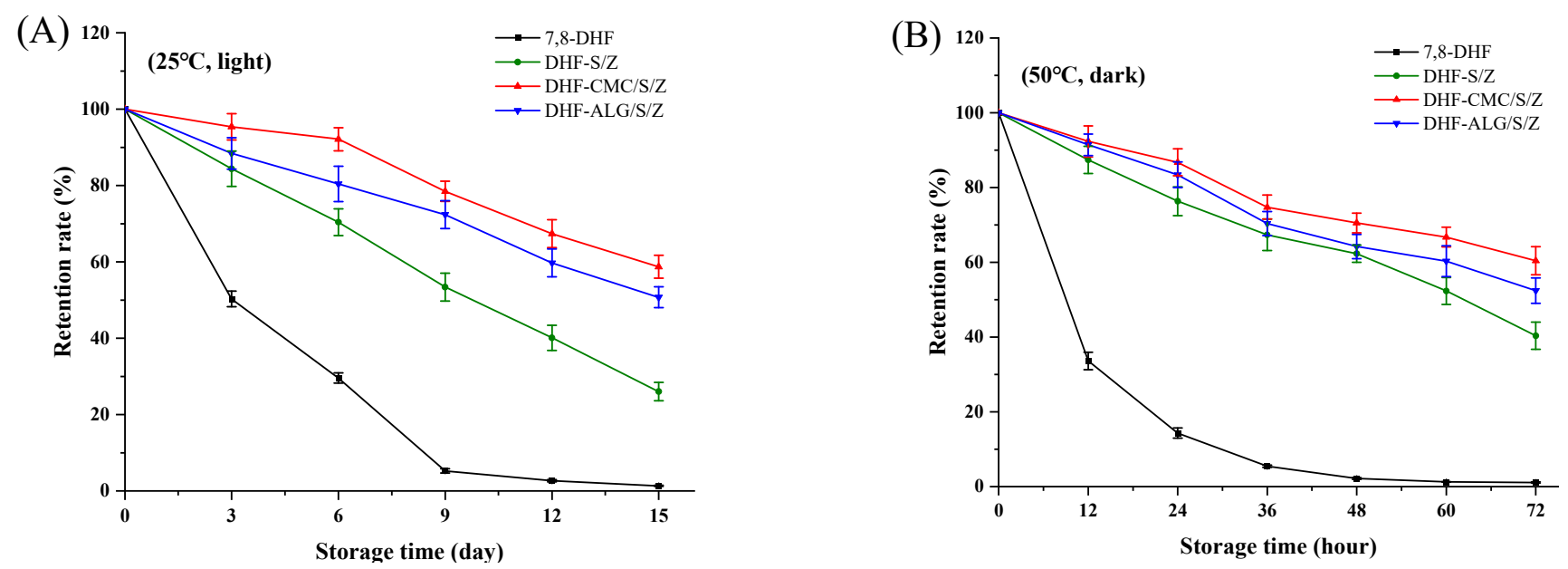

Figure 8. Storage stability of free 7,8-DHF, DHF-S/Z, DHF-CMC/S/Z and DHF-ALG/S/Z at $25^{\circ} \mathrm{C}$ under light (A), at $50{ }^{\circ} \mathrm{C}$ under dark (B).

\subsection{In Vitro Simulated Gastrointestinal Digestion}

A gastrointestinal tract (GIT) model was applied to study the digestive fate and bioaccessibility of 7,8-DHF in different formulations. Particle size changes were monitored at a specific digestion time (30,60,120, and $180 \mathrm{~min})$, and the results were presented in (Figure 9A). The mean particle size of DHF-S/Z was significantly increased after $60 \mathrm{~min}$ SGF digestion $(p<0.05)$. This finding was possibly due to the fact that the $S / Z$ were exposed to ionic strength along with low $\mathrm{pH}$ and partially digestion via pepsase. The low $\mathrm{pH}$ and ionic strength exposure likely weakened electrostatic repulsion forces among the nanoparticles [52]. Particle size reduction in DHF-S/Z post-SIF-exposure was attributed to the fact that SIF contains bile salt with strong emulsifying ability. Bile salt can bind many biopolymer molecules and induce bridging flocculation [21]. The particle size of DHF-CMC/S/Z was increased after exposure to the stomach phase but remained relatively constant during incubation in SIF. This behavior suggested that the existence of CMC strengthened intestinal stability of DHF-CMC/S/Z. However, the particle size of DHFALG/S/Z remained fairly steady throughout simulated GIT, only showing a large increase at $30 \mathrm{~min}$ during the SGF incubation. The different influence of polysaccharide type (CMC or ALG) on gastrointestinal fate of colloidal carriers can be attributed to different molecular characteristics. FE-SEM microscopic observation further confirmed that exposure to simulated gastrointestinal conditions had a significant effect on the morphology of the 7,8DHF-loaded nano-complexes (Figure 9C). For DHF-S/Z, irregular shapes have developed after they were added into the SGF and SIF, similar to an anomalous sheet structure. This shape acquisition was due to irregular aggregates appearing after SGF digestion. Furthermore, DHF-CMC/S/Z and DHF-ALG/S/Z exhibited a spherical shape. After gastrointestinal digestion, the nanoparticles possessed a relatively spherical morphology, resembling the cross-linked structure of large nanoparticles. Overall, CMC and ALG effectively protected the stability of DHF-S/Z nanoparticles through the GIT. Particularly, ALG performed extremely well. 

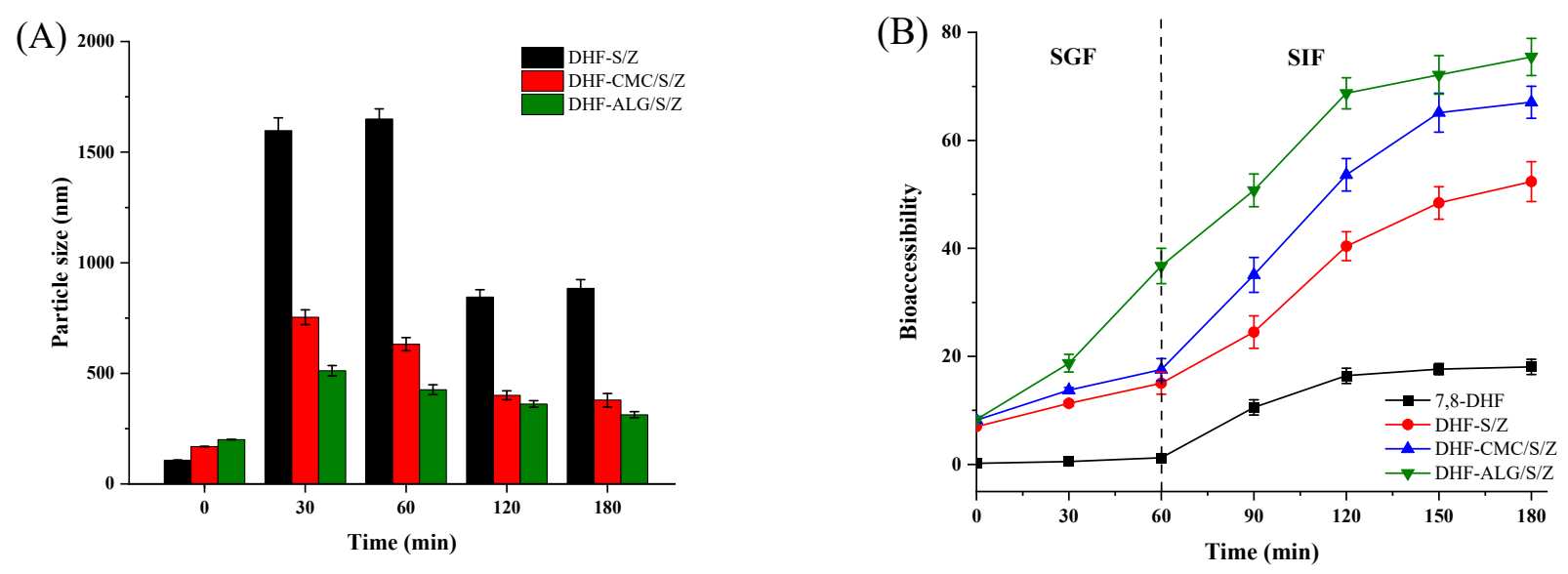

(C)
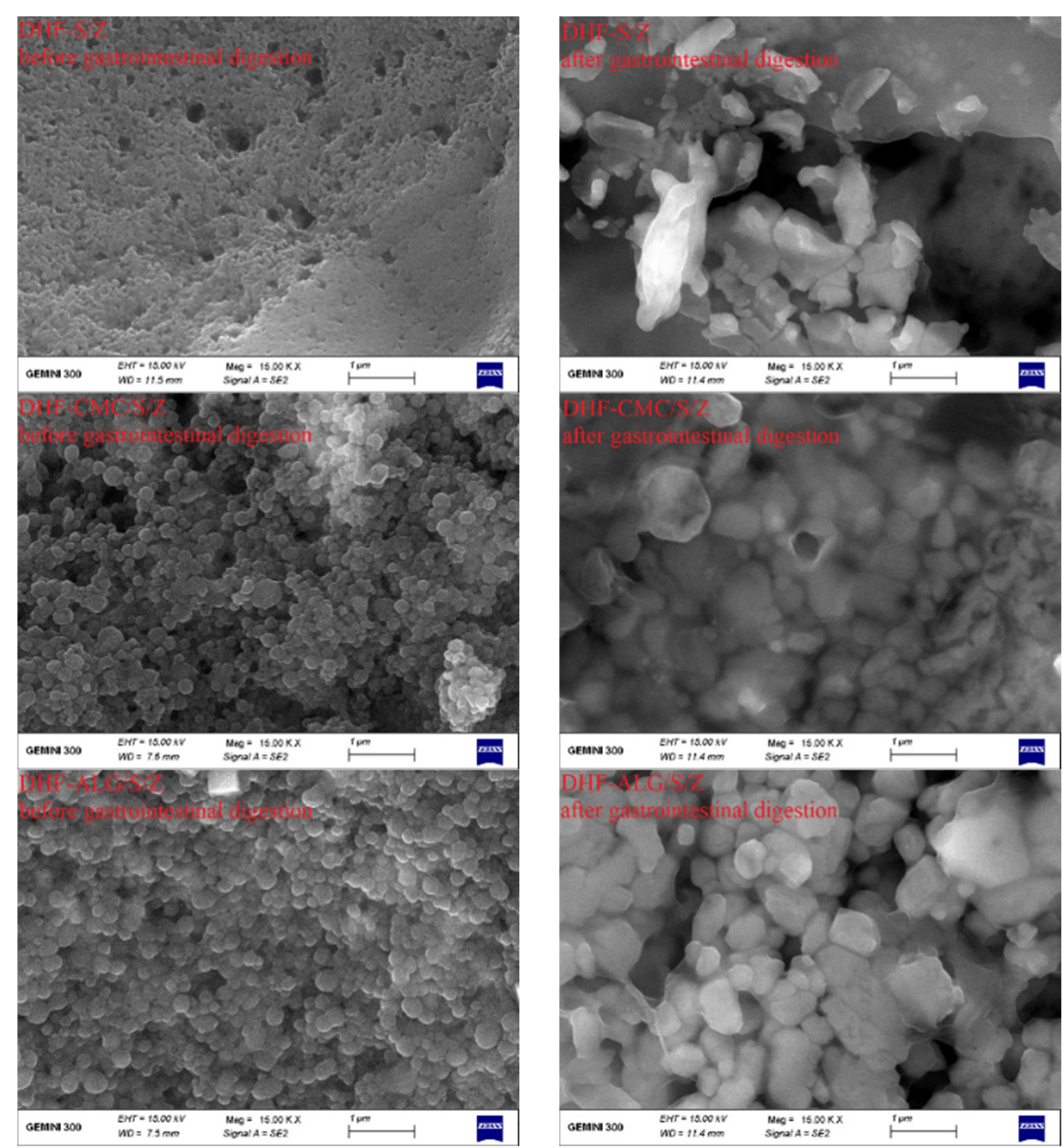

Figure 9. Influence of in vitro digestion time on the particle size (A) and bio-accessibility of DHF-S/Z, DHF-CMC/S/Z, and DHF-ALG/S/Z (B), FE-SEM images of DHF-S/Z, DHF-CMC/S/Z, and DHF-ALG/S/Z, 15,000× magnification times (C).

After being exposed to simulated gastrointestinal conditions, 7,8-DHF bioaccessibility was measured after centrifugation and collection of micelle phases. As shown in Figure $9 \mathrm{C}$, the $\mathrm{S} / \mathrm{Z}$ was broken down when exposed to SGF digestion, and the core of 7,8-DHF was released, resulting in a low bioaccessibility. The bioaccessibility of DHF-S/Z gradually increased in SIF digestion, primarily due to the emulsibility of bile salts and sophorolipids. Another reason for this increase was the binding of hydrolyzed peptides of zein protein [33]. In the presence of CMC, low levels of bioaccessibility were exhibited in SGF digestion. This behavior was introduced by the vulnerability of CMC to acid, 
pepsin enzymes, and the alkali ions of SGF juices [53]. However, the bioaccessibility of DHF-CMC/S/Z was higher than that of DHF-S/Z, indicating a synergistic effect existed among $\mathrm{CMC}$, sophorolipid and zein on controlling the release of 7,8-DHF. Furthermore, the presence of ALG further increased bioaccessibility to $75.46 \%$ (Figure 9B), showing the highest bioaccessibility of 7,8-DHF after GIT digestion compared to DHF-CMC/S/Z and DHF-S/Z. These results indicated that ALG increased the solubilization capacity of mixed micelles in small intestine fluids. Collectively, our results demonstrated that encapsulating 7,8-DHF in polysaccharide coated S/Z nanoparticles can promote an appreciable increase in its in vitro bioaccessibility.

\section{Conclusions}

In the present study, we compared the performance of ternary nanoparticles in the existence of two selected polysaccharides (CMC and ALG) for encapsulation of bioactive 7,8-DHF but with low bioavailability. CMC/S/Z exhibited lower PDI, particle size and turbidity, but higher zeta potential and loading capacity compared to ALG/S/Z. Furthermore, both polysaccharides supplementations promoted the EE value of 7,8-DHF in $\mathrm{S} / \mathrm{Z}$, especially CMC. Invitation of polysaccharides displayed positive effects on the formation and physical stability ( $\mathrm{pH}$ and ionic strength stability) of ternary complexes. The formation of ternary complexes mainly occurred via hydrophobic effects, hydrogen bonding and electrostatic interactions. More significantly, compared to $S / Z, A L G / S / Z$, and $\mathrm{CMC} / \mathrm{S} / \mathrm{Z}$ obviously enhanced the storage stability and in vitro bioaccessibility of 7,8-DHF. CMC/S/Z possessed a higher storage stability for 7,8-DHF. In contrast, ALG/S/Z had a better in vitro bioaccessibility of 7,8-DHF. Collectively, the results of this study indicate that selected polysaccharides containing composite nanoparticles are efficient at encapsulating, retaining, and delivering 7,8-DHF, and might therefore be utilized in dietary supplements and functional foods. Future work will focus on the applicability of DHF-ALG/S/Z and DHF-CMC/S/Z in complex water-phase beverage systems. Besides, the transepithelial transport mechanism of DHF-ALG/S/Z in an Caco-2 cell model, and in vivo pharmacokinetic studies in rat will be also studies.

Supplementary Materials: The following are available online at https:/ / www.mdpi.com/article/10 .3390 / foods10112629/s1, Figure S1: The chemical structure of 7,8-DHF. Figure S2: Size distributions of nanoparticles with different zein to polysaccharide mass ratios, CMC/S/Z (A), ALG/S/Z (B). Figure S3: Size distributions of nanoparticles with different zein to polysaccharide mass ratios at $\mathrm{pH}$ $=4, \mathrm{CMC} / \mathrm{S} / \mathrm{Z}$ (A), ALG/S/Z (B). Figure S4: The photograph of each colloidal particle at different $\mathrm{pH}$ conditions. Figure S5: The photograph of each colloidal particle at different $\mathrm{NaCl}$ concentrations.

Author Contributions: Conceptualization, methodology, resources, funding acquisition and writingoriginal draft preparation, Y.C.; software and formal analysis, J.P. and X.G.; investigation and data curation, L.W. and Y.S.; writing-review and editing, D.W.; project administration, Y.W.; validation and supervision, G.X. All authors have read and agreed to the published version of the manuscript.

Funding: This research was funded by the Zhejiang Province Public Welfare Technology Application Research Project -National Cooperation Project (grant number LGJ21C20001), Fund of Key Laboratory of Aquatic Product Processing, Ministry of Agriculture and Rural Affairs, China (grant number NYJG202104), Zhejiang Province Xinmiao Talents Program (grant number 2021R403035) and College students' Innovation and Entrepreneurship Training program of China (grant number 2021052 and 2021011).

Institutional Review Board Statement: Not applicable.

Informed Consent Statement: Not applicable.

Data Availability Statement: Not applicable.

Acknowledgments: All authors are thankful to their representative universities/institutes for the support and services used in this study.

Conflicts of Interest: The authors declare no conflict of interest. 


\section{References}

1. Andero, R.; Ressler, K.J. Fear extinction and bdnf: Translating animal models of ptsd to the clinic. Genes Brain Behav. 2012, 11, 503-512. [CrossRef] [PubMed]

2. Colombo, P.S.; Flamini, G.; Christodoulou, M.S.; Rodondi, G.; Vitalini, S.; Passarella, D.; Fico, G. Farinose alpine primula species: Phytochemical and morphological investigations. Phytochemistry 2014, 98, 151-159. [CrossRef] [PubMed]

3. Jang, S.; Liu, X.; Yepes, M.; Shepherd, K.R.; Miller, G.W.; Liu, Y.; Wilson, W.D.; Xiao, G.; Blanchi, B.; Sun, Y.E. A selective trkb agonist with potent neurotrophic activities by 7,8-dihydroxyflavone. Proc. Natl. Acad. Sci. USA 2010, 107, 2687-2692. [CrossRef] [PubMed]

4. $\quad$ Li, X.H.; Dai, C.F.; Chen, L.; Zhou, W.T.; Han, H.L.; Dong, Z.F. 7,8-dihydroxyflavone ameliorates motor deficits via suppressing $\alpha$-synuclein expression and oxidative stress in the mptp-induced mouse model of parkinson's disease. CNS Neurosci. Ther. 2016, 22, 617-624. [CrossRef] [PubMed]

5. Aytan, N.; Choi, J.; Carreras, I.; Crabtree, L.; Nguyen, B.; Lehar, M.; Blusztajn, J.K.; Jenkins, B.G.; Dedeoglu, A. Protective effects of 7,8-dihydroxyflavone on neuropathological and neurochemical changes in a mouse model of alzheimer's disease. Eur. J. Pharmacol. 2018, 828, 9-17. [CrossRef]

6. Chan, C.B.; Tse, M.C.L.; Liu, X.; Zhang, S.; Schmidt, R.; Otten, R.; Liu, L.; Ye, K. Activation of muscular trkb by its small molecular agonist 7,8-dihydroxyflavone sex-dependently regulates energy metabolism in diet-induced obese mice. Chem. Biol. 2015, 22, 355-368. [CrossRef]

7. Chen, C.; Wang, Z.; Zhang, Z.; Liu, X.; Kang, S.S.; Zhang, Y.; Ye, K. The prodrug of 7,8-dihydroxyflavone development and therapeutic efficacy for treating alzheimer's disease. Proc. Natl. Acad. Sci. USA 2018, 115, 578-583. [CrossRef]

8. Zhang, J.; Yao, W.; Dong, C.; Yang, C.; Ren, Q.; Ma, M.; Han, M.; Hashimoto, K. Comparison of ketamine, 7,8-dihydroxyflavone, and ana-12 antidepressant effects in the social defeat stress model of depression. Psychopharmacology 2015, 232, 4325-4335. [CrossRef]

9. Liu, C.; Chan, C.B.; Ye, K. 7,8-dihydroxyflavone, a small molecular trkb agonist, is useful for treating various bdnf-implicated human disorders. Transl. Neurodegener. 2016, 5, 1-9. [CrossRef]

10. Chen, Y.; Xue, F.; Xia, G.; Zhao, Z.; Chen, C.; Li, Y.; Zhang, Y. Transepithelial transport mechanisms of 7,8-dihydroxyflavone, a small molecular trkb receptor agonist, in human intestinal caco-2 cells. Food Funct. 2019, 10, 5215-5227. [CrossRef]

11. Chen, Y.; Xia, G.; Zhao, Z.; Xue, F.; Gu, Y.; Chen, C.; Zhang, Y. 7,8-dihydroxyflavone nano-liposomes decorated by crosslinked and glycosylated lactoferrin: Storage stability, antioxidant activity, in vitro release, gastrointestinal digestion and transport in caco-2 cell monolayers. J. Funct. Foods 2020, 65, 103742. [CrossRef]

12. Behjati, J.; Yazdanpanah, S. Nanoemulsion and emulsion vitamin d3 fortified edible film based on quince seed gum. Carbohyd. Polym. 2021, 262, 117948. [CrossRef]

13. Niu, F.; Hu, D.; Gu, F.; Du, Y.; Zhang, B.; Ma, S.; Pan, W. Preparation of ultra-long stable ovalbumin/sodium carboxymethylcellulose nanoparticle and loading properties of curcumin. Carbohyd. Polym. 2021, 271, 118451. [CrossRef]

14. Zhou, W.; Zhang, Y.; Li, R.; Peng, S.; Ruan, R.; Li, J.; Liu, W. Fabrication of caseinate stabilized thymol nanosuspensions via the ph-driven method: Enhancement in water solubility of thymol. Foods 2021, 10, 1074. [CrossRef] [PubMed]

15. Gao, J.; Liu, C.; Shi, J.; Ni, F.; Shen, Q.; Xie, H.; Wang, K.; Lei, Q.; Fang, W.; Ren, G. The regulation of sodium alginate on the stability of ovalbumin-pectin complexes for vd3 encapsulation and in vitro simulated gastrointestinal digestion study. Food Res. Int. 2021, 140, 110011. [CrossRef] [PubMed]

16. Zhang, J.; Liu, Y.; Liu, X.; Li, Y.; Yin, X.; Subirade, M.; Zhou, P.; Liang, L. The folic acid/ $\beta$-casein complex: Characteristics and physicochemical implications. Food Res. Int. 2014, 57, 162-167. [CrossRef]

17. Chen, S.; Ma, Y.; Dai, L.; Liao, W.; Zhang, L.; Liu, J.; Gao, Y. Fabrication, characterization, stability and re-dispersibility of curcumin-loaded gliadin-rhamnolipid composite nanoparticles using ph-driven method. Food Hydrocolloid. 2021, 118, 106758. [CrossRef]

18. Xiao, Y.; Ho, C.; Chen, Y.; Wang, Y.; Wei, Z.; Dong, M.; Huang, Q. Synthesis, characterization, and evaluation of genistein-loaded zein/carboxymethyl chitosan nanoparticles with improved water dispersibility, enhanced antioxidant activity, and controlled release property. Foods 2020, 9, 1604. [CrossRef] [PubMed]

19. Li, Y.; Xu, G.; Li, W.; Lv, L.; Zhang, Q. The role of ultrasound in the preparation of zein nanoparticles/flaxseed gum complexes for the stabilization of pickering emulsion. Foods 2021, 10, 1990. [CrossRef] [PubMed]

20. Wei, Y.; Wang, C.; Liu, X.; Mackie, A.; Zhang, L.; Liu, J.; Mao, L.; Yuan, F.; Gao, Y. Impact of microfluidization and thermal treatment on the structure, stability and in vitro digestion of curcumin loaded zein-propylene glycol alginate complex nanoparticles. Food Res. Int. 2020, 138, 109817. [CrossRef]

21. Dai, L.; Wei, Y.; Sun, C.; Mao, L.; Mcclements, D.J.; Gao, Y. Development of protein-polysaccharide-surfactant ternary complex particles as delivery vehicles for curcumin. Food Hydrocolloid. 2018, 85, 75-85. [CrossRef]

22. Wei, Y.; Zhang, L.; Yu, Z.; Lin, K.; Yang, S.; Dai, L.; Liu, J.; Mao, L.; Yuan, F.; Gao, Y. Enhanced stability, structural characterization and simulated gastrointestinal digestion of coenzyme $Q_{10}$ loaded ternary nanoparticles. Food Hydrocolloid. 2019, 94, 333-344. [CrossRef]

23. Zhang, H.; Fu, Y.; Xu, Y.; Niu, F.; Li, Z.; Ba, C.; Jin, B.; Chen, G.; Li, X. One-step assembly of zein/caseinate/alginate nanoparticles for encapsulation and improved bioaccessibility of propolis. Food Funct. 2019, 10, 635-645. [CrossRef] [PubMed] 
24. Liu, Q.; Jing, Y.; Han, C.; Zhang, H.; Tian, Y. Encapsulation of curcumin in zein/caseinate/sodium alginate nanoparticles with improved physicochemical and controlled release properties. Food Hydrocolloid. 2019, 93, 432-442. [CrossRef]

25. Chen, Y.; Xia, G.; Zhao, Z.; Xue, F.; Chen, C.; Zhang, Y. Formation, structural characterization, stability and in vitro bioaccessibility of 7,8-dihydroxyflavone loaded zein-/sophorolipid composite nanoparticles: Effect of sophorolipid under two blending sequences. Food Funct. 2020, 11, 1810-1825. [CrossRef] [PubMed]

26. Afshar, M.; Dini, G.; Vaezifar, S.; Mehdikhani, M.; Movahedi, B. Preparation and characterization of sodium alginate/polyvinyl alcohol hydrogel containing drug-loaded chitosan nanoparticles as a drug delivery system. J. Drug Deliv. Sci. Technol. 2020, 56, 101530. [CrossRef]

27. Sheng, Y.; Gao, J.; Yin, Z.; Kang, J.; Kong, Y. Dual-drug delivery system based on the hydrogels of alginate and sodium carboxymethyl cellulose for colorectal cancer treatment. Carbohyd. Polym. 2021, 269, 118325. [CrossRef]

28. Yuan, Y.; Li, H.; Liu, C.; Zhang, S.; Xu, Y.; Wang, D. Fabrication and characterization of lutein-loaded nanoparticles based on zein and sophorolipid: Enhancement of water solubility, stability, and bioaccessibility. J. Agric. Food Chem. 2019, 67, 11977-11985. [CrossRef]

29. Wei, Y.; Yu, Z.; Lin, K.; Sun, C.; Dai, L.; Yang, S.; Mao, L.; Yuan, F.; Gao, Y. Fabrication and characterization of resveratrol loaded zein-propylene glycol alginate-rhamnolipid composite nanoparticles: Physicochemical stability, formation mechanism and in vitro digestion. Food Hydrocolloid. 2019, 95, 336-348. [CrossRef]

30. Dai, L.; Sun, C.; Wei, Y.; Zhan, X.; Mao, L.; Gao, Y. Formation and characterization of zein-propylene glycol alginate-surfactant ternary complexes: Effect of surfactant type. Food Chem. 2018, 258, 321-330. [CrossRef]

31. Patel, A.R.; Ten-Hoorn, J.S.; Hazekamp, J.; Blijdenstein, T.B.; Velikov, K.P. Colloidal complexation of a macromolecule with a small molecular weight natural polyphenol: Implications in modulating polymer functionalities. Soft Matter 2013, 9, 1428-1436. [CrossRef]

32. Peng, S.; Li, Z.; Zou, L.; Liu, W.; Liu, C.; Mcclements, D.J. Enhancement of curcumin bioavailability by encapsulation in sophorolipid-coated nanoparticles: An in vitro and in vivo study. J. Agric. Food Chem. 2018, 66, 1488-1497. [CrossRef]

33. Yao, K.; Chen, W.; Song, F.; Mcclements, D.J.; Hu, K. Tailoring zein nanoparticle functionality using biopolymer coatings: Impact on curcumin bioaccessibility and antioxidant capacity under simulated gastrointestinal conditions. Food Hydrocolloid. 2018, 79, 262-272. [CrossRef]

34. Hu, K.; Mcclements, D.J. Fabrication of biopolymer nanoparticles by antisolvent precipitation and electrostatic deposition: Zein-alginate core/shell nanoparticles. Food Hydrocolloid. 2015, 44, 101-108. [CrossRef]

35. Dai, L.; Li, R.; Wei, Y.; Sun, C.; Mao, L.; Gao, Y. Fabrication of zein and rhamnolipid complex nanoparticles to enhance the stability and in vitro release of curcumin. Food Hydrocolloid. 2018, 77, 617-628. [CrossRef]

36. Yuan, Y.; Li, H.; Liu, C.; Zhu, J.; Xu, Y.; Zhang, S.; Fan, M.; Zhang, D.; Zhang, Y.; Zhang, Z. Fabrication of stable zein nanoparticles by chondroitin sulfate deposition based on antisolvent precipitation method. Int. J. Biol. Macromol. 2019, 139, 30-39. [CrossRef]

37. Sun, C.; Dai, L.; Gao, Y. Binary complex based on zein and propylene glycol alginate for delivery of quercetagetin. Biomacromolecules 2016, 17, 3973-3985. [CrossRef] [PubMed]

38. Zhao, W.; Yuan, P.; She, X.; Xia, Y.; Komarneni, S.; Xi, K.; Che, Y.; Yao, X.; Yang, D. Sustainable seaweed-based one-dimensional (1d) nanofibers as high-performance electrocatalysts for fuel cells. J. Mater. Chem. A 2015, 3, 14188-14194. [CrossRef]

39. Xu, Y.; Liu, L. Structural and functional properties of soy protein isolates modified by soy soluble polysaccharides. J. Agric. Food Chem. 2016, 64, 7275-7284. [CrossRef] [PubMed]

40. Cerqueira, M.A.; Souza, B.W.; Teixeira, J.A.; Vicente, A.A. Effect of glycerol and corn oil on physicochemical properties of polysaccharide films-A comparative study. Food Hydrocolloid. 2012, 27, 175-184. [CrossRef]

41. Luo, Y.; Zhang, B.; Whent, M.; Yu, L.L.; Wang, Q. Preparation and characterization of zein/chitosan complex for encapsulation of $\alpha$-tocopherol, and its in vitro controlled release study. Colloids Surf. B Biointerfaces 2011, 85, 145-152. [CrossRef] [PubMed]

42. Liu, F.; Ma, C.; Mcclements, D.J.; Gao, Y. A comparative study of covalent and non-covalent interactions between zein and polyphenols in ethanol-water solution. Food Hydrocolloid. 2017, 63, 625-634. [CrossRef]

43. Chen, Y.; Zhao, Z.; Xia, G.; Xue, F.; Chen, C.; Zhang, Y. Fabrication and characterization of zein/lactoferrin composite nanoparticles for encapsulating 7,8-dihydroxyflavone: Enhancement of stability, water solubility and bioaccessibility. Int. J. Biol. Macromol. 2020, 146, 179-192. [CrossRef]

44. Liu, E.; Su, Z.; Yang, C.; Ji, Y.; Liu, B.; Meng, X. Fabrication, characterization and properties of dha-loaded nanoparticles based on zein and plga. Food Chem. 2021, 360, 129957. [CrossRef]

45. Lancelot, E.; Fontaine, J.; Grua-Priol, J.; Assaf, A.; Thouand, G.; Le-Bail, A. Study of structural changes of gluten proteins during bread dough mixing by raman spectroscopy. Food Chem. 2021, 358, 129916. [CrossRef]

46. Narwal, S.; Kumar, A.; Chaudhary, M.; Budhwar, V. Formulation of eutectic mixture of curcumin with salicylic acid for improving its dissolution profile. Res. J. Pharm. Technol. 2021, 14, 1875-1879.

47. Jiang, F.; Yang, L.; Wang, S.; Ying, X.; Ling, J.; Ouyang, X.K. Fabrication and characterization of zein-alginate oligosaccharide complex nanoparticles as delivery vehicles of curcumin. J. Mol. Liq. 2021, 342, 116937. [CrossRef]

48. Wang, X.; Li, M.; Liu, F.; Peng, F.; Li, F.; Lou, X.; Jin, Y.; Wang, J.; Xu, H. Fabrication and characterization of zein-tea polyphenolspectin ternary complex nanoparticles as an effective hyperoside delivery system: Formation mechanism, physicochemical stability, and in vitro release property. Food Chem. 2021, 364, 130335. [CrossRef] [PubMed] 
49. Sun, C.; Dai, L.; Gao, Y. Interaction and formation mechanism of binary complex between zein and propylene glycol alginate. Carbohyd. Polym. 2017, 157, 1638-1649. [CrossRef]

50. Wang, W.; Liu, F.; Gao, Y. Quercetagetin loaded in soy protein isolate-K-carrageenan complex: Fabrication mechanism and protective effect. Food Res. Int. 2016, 83, 31-40. [CrossRef]

51. Liang, H.; Zhou, B.; He, L.; An, Y.; Lin, L.; Li, Y.; Liu, S.; Chen, Y.; Li, B. Fabrication of zein/quaternized chitosan nanoparticles for the encapsulation and protection of curcumin. RSC Adv. 2015, 5, 13891-13900. [CrossRef]

52. Chen, S.; Han, Y.; Huang, J.; Dai, L.; Du, J.; Mcclements, D.J.; Mao, L.; Liu, J.; Gao, Y. Fabrication and characterization of layer-by-layer composite nanoparticles based on zein and hyaluronic acid for codelivery of curcumin and quercetagetin. ACS Appl. Mater. Inter. 2019, 11, 16922-16933. [CrossRef] [PubMed]

53. Parris, N.; Cooke, P.H.; Hicks, K.B. Encapsulation of essential oils in zein nanospherical particles. J. Agric. Food Chem. 2005, 53, 4788-4792. [CrossRef] [PubMed] 